\title{
Wild edible plants in Yeşilli (Mardin-Turkey), a multicultural area
}

\author{
Yeter Yeşil ${ }^{*} \mathbb{D}$, Mahmut Çelik and Bahattin Yılmaz
}

\begin{abstract}
Background: The Yeşilli district (Mardin) is located in the southeastern of Turkey and hosts different cultures. The objective of this study was to record the traditional knowledge of wild edible plants used by indigenous people in Yeşilli, where no ethnobotanical studies have been conducted previously.

Methods: An ethnobotanical study was carried out in Yeşilli district in March 2017-March 2019 to document the traditional knowledge of wild edible plants. The data were collected by interviewing 62 informants. Additionally, the data were analysed based on the cultural importance index $(\mathrm{Cl})$ and factor informant consensus $\left(F_{\text {ic }}\right)$ to determine the cultural significance of wild edible plants and knowledge of wild edible plants among the informants.

Results: We documented 74 wild edible taxa belonging to 31 families and 57 genera in the present study. The richness of the wild edible taxa was highest for vegetables (46 taxa), followed by medicinal plants ( 17 taxa) and fruit (14 taxa). The most important families were Asteraceae (ten taxa), Rosaceae (seven taxa) and Fabaceae (six taxa). The most culturally important taxa (based on the $\mathrm{Cl}$ index) were Ficus carica subsp. carica, Lepidium draba, Anchusa strigosa, Rhus coriaria, Glycyrrhiza glabra, Sinapis alba, Gundelia tournefortii, Notobasis syriaca, Onopordum carduchorum, Malva neglecta, Mentha longifolia, Juglans regia and Urtica dioica. The maximum number of use reports was recorded for vegetables (1011). The factor informant consensus index $\left(F_{i c}\right)$ varied between 0.95 and 0.98 for preserved vegetables, beverages and spices and processed fruits have the highest $F_{i c}(0.99)$. We reported for the first time the ethnobotanical usage of 12 taxa as food. We also recorded the use of Allium wendelboanum, an endemic species in the study area.

Conclusion: The obtained data were compared with data from other wild edible and ethnobotanical studies conducted in Turkey and particularly those conducted in eastern Turkey. Furthermore, the data were compared with data from studies conducted in the bordering countries of Iraq and Armenia. The present study reflects the cultural diversity of the region, and it is necessary to conduct more studies since it is thought that this diversity will contribute to the economy. This study will enable the traditional use of wild plants as food sources to be passed on to future generations.
\end{abstract}

Keywords: Ethnobotany, Wild edible plants, Cultural Importance index, Yeşilli, Mardin

\section{Introduction}

Traditional knowledge of plants and their uses is the result of thousands of years of experience. The relevance of this knowledge to the increased daily living standards of rural populations as well as to decisions regarding the sustainable use of plant resources has frequently been noted [1].

* Correspondence: yeteryesil@yahoo.com

Faculty of Pharmacy, Department of Pharmaceutical Botany, Istanbul University, Fatih, 34116 Istanbul, Turkey
Wild edible plants are defined as non-cultivated and non-domesticated plants [2]. Interest in the use of wild food sources has increased in recent years. Valuable nutritional supplements, including trace elements, vitamins and minerals, are acquired from many wild plants [3]. These supplements are known as 'nutraceuticals', and their importance has recently been highlighted [2].

In recent years, detailed information about wild food plants in Europe has been reviewed [2-4]. According to these studies, most of the plants that were previously used as food are used rarely or not at all today. However,

(c) The Author(s). 2019 Open Access This article is distributed under the terms of the Creative Commons Attribution 4.0 International License (http://creativecommons.org/licenses/by/4.0/), which permits unrestricted use, distribution, and 
while the traditional use of wild edible plants has greatly reduced due to socio-economic and ecological changes, wild plants are becoming a part of a new way of thinking about food: they are very important in healthy food, food safety and slow food movements. Ethnobotanical studies conducted in various parts of Europe have prevented the use of wild plants as food [5-12].

The Turkish flora comprises 8796 species, listed in 'Flora of Turkey' [13-15]. Additionally, according to 'Illustrated Flora of Turkey' [16-18], the most recent checklist [19] and publications about Turkish flora include 11,700 taxa. The endemism rate is $34 \%$ [17]. The Anatolian people, who have been living in this area for centuries have benefited from this rich diversity of plants primarily for food, medicine and other purposes [20]. However, systematic ethnobotanical studies only began in the mid1990s [21] in Turkey. In rural areas, the people acquire the plants they need by gathering them from the surrounding mountains and forests [22-24].

Numerous studies on ethnobotanical and edible plants have been conducted in various regions of Turkey [25-94]. However, the southeastern region of Turkey, which was the focus of the present research, has been the subject of only a limited number of ethnobotanical studies [26-28, 31, 52, 60]. Several previous ethnobotanical studies have described the traditional knowledge of plants close to the present study area [31, 60]. Additionally, ethnobotanical studies have been conducted in nearby regions with the same cultural structure as the Yeșilli district [95-99].

Mardin province is a region within the boundaries of the region called ancient Mesopotamia that has hosted many civilisations. Since the beginning of history, many people and religious communities have lived together in this region. The different denominations of Muslims, Christians, Jews and Yezidis who believe in Melek Taus and the heliolatry Shams make up the majority of Mardin city. The Sun Temple in the ancient city of Dara, the Temple of Zarathustra where Deyrulzafaran Monastery was built and the Tur Abdin region called Abidler Dagi are important places that show that the region has been a religious centre for thousands of years [100]. The multicultural structure of Mardin has continued to the present day; Kurds, Assyrians, Arabs and Yezidis currently live together in this area. For this reason, at least three languages are spoken in daily life $[100,101]$.

The Yeșilli district of Mardin city, which is home to a portion of Mardin's diverse culture, consists of nine rural and five urban settlements. The cultural diversity of the district is reflected in the local ethnobotanical knowledge. Although agriculture has declined in many regions in Turkey, it continues in Yeşilli and because of this, the population is dense in rural areas, and there have not been botanical studies in this area. The focus of the present study was to identify wild edible plants, rather than agricultural plants. Yeşilli was selected because of its mix of cultures and the fact that no ethnobotanical or floristic studies had preciously been conducted in this region. We focused on (1) identifying edible plant use, (2) determining new uses of wild edible plants and evaluating our findings in terms of cultural ethnobotany by comparing with previous ethnobotanical studies and (3) transferring this knowledge to future generations and offering alternative sustainable sources of food.

\section{Materials and methods \\ Study area}

Yeșilli (formerly Rișmil) is a district of Mardin city, which is located in the southeast region of Turkey $\left(37^{\circ}\right.$ $20^{\prime} 20.95^{\prime \prime} \mathrm{N}$ and $40^{\circ} 49^{\prime} 20.81^{\prime \prime} \mathrm{E}$ ) (Fig. 1). This area lies at an altitude of approximately $2696 \mathrm{ft}$ a.s.l. Yeşilli district consists of nine rural settlements and five urban settlements.

Yeşilli has a transitional climate between the continental climate and the Mediterranean climate. The summers are hot, and the winters are cold. Most rainfall occurs in winter and spring [102]. The highest annual temperature is $38.5{ }^{\circ} \mathrm{C}$ (August), and the lowest is $0{ }^{\circ} \mathrm{C}$ (January) [103]. This area belongs to the Irano-Turanian Plant Geography Region and falls within the B8 grid square according to the grid classification system developed by Davis [13]. The area is covered with small oak forests and maquis. Quercus coccifera L., Q. brantii Lindl., Olea europaea Lindl., Pistacia terebinthus L., Paliurus spina-christii P. Mill. and Juniperus oxycedrus L. are among the important maquis species in this area [17]. The district is the greenest area in Mardin city.

\section{Socio-economic profile}

Yeșilli district is so named because horticulture and agriculture are highly developed in this region; 'Yeşilli' means 'extensive greenery'. Almond and olive gardens and vineyards can be found in Bülbül, Dereyanı, Sancar and Uzunköy, as well as several other villages (Fig. 2). In other villages, sheep and goat breeding is common. People who live in Bülbül village prepare wine and olive oil to sell and trade almonds, olives and grapes. The inhabitants of other villages trade almonds, olives and grapes. In terms of agriculture, wheat, barley, lentils and chickpeas are cultivated.

Christians and Muslims have lived together for thousands of years in Yeșilli. Furthermore, four different languages, Turkish (official language), Kurdish, Arabic and Syriac, are spoken in this region. The population of this region is 16877 according to the address-based census of 2013 [102]. Yeşilli district has one Christian village, Bülbül. The inhabitants of this village are Assyrians, but they have forgotten their language; only those who study in the Monastery of Deyrulzafaran (Mardin) can write, 


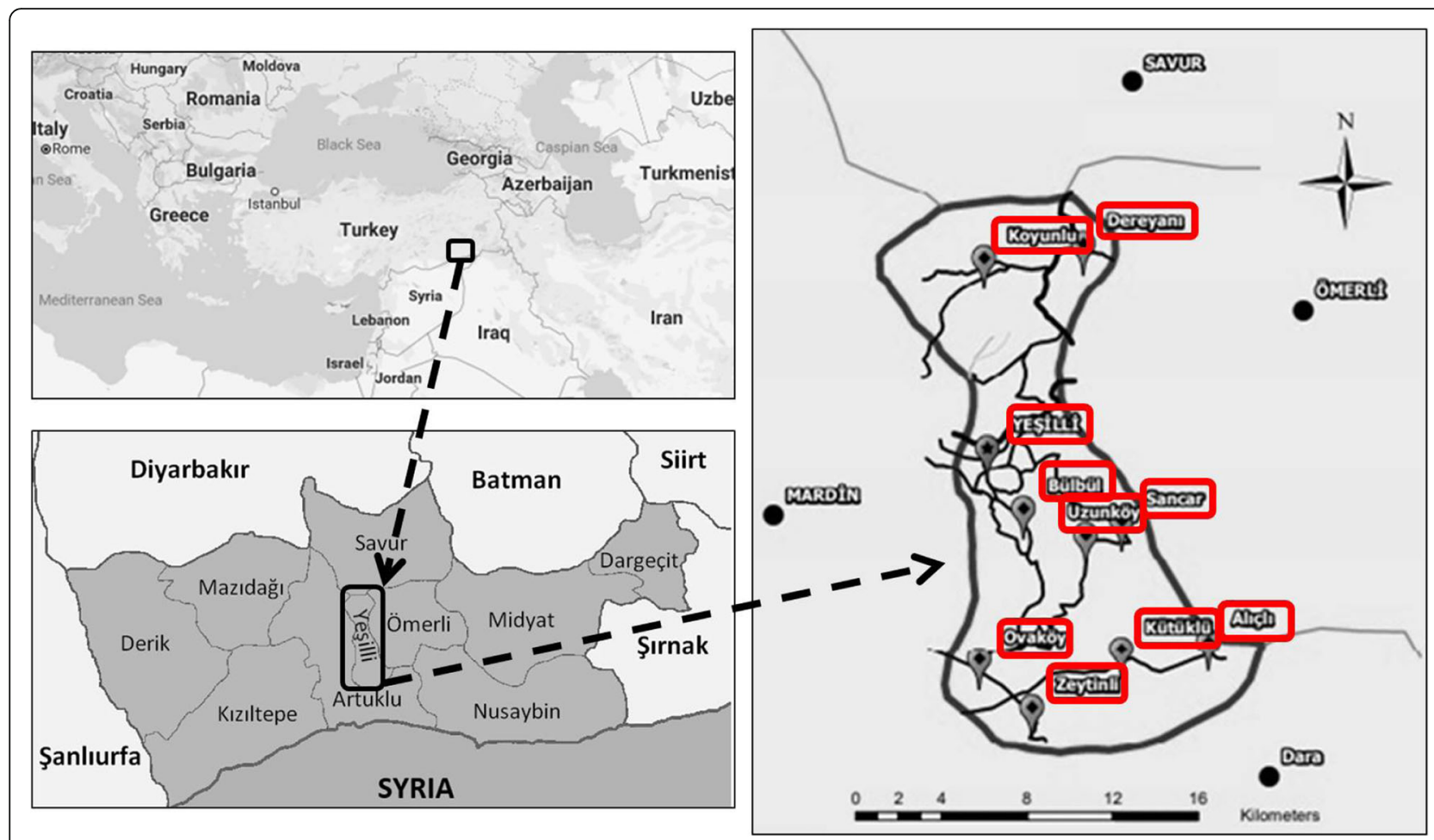

Fig. 1 Map of Yeşilli and Districts. Provinces: Koyunlu, Dereyanı, Yeşilli city center, Bülbül, Sancar, Uzunköy, Alıçlı, Kütüklü, Ovaköy and Zeytinli

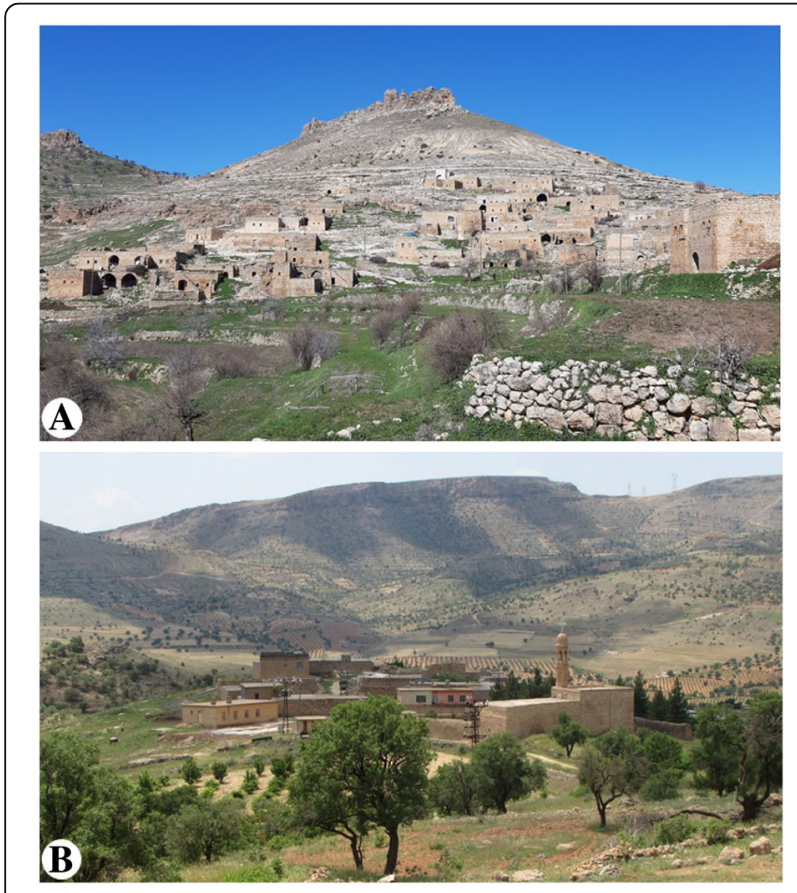

Fig. 2 The villages of Yeşilli. a Sancar village. b Bülbül village read and speak the language. Twenty percent of the population can speak Syriac, and the older members of the population cannot speak Turkish at all. People speak Arabic, Kurdish and Syriac in their daily lives. Kurdish people live in the other eight villages, and they speak Kurdish, Turkish and sometimes Arabic. The majority of the population in the district centre is Arabic and speaks Arabic, Turkish and Kurdish.

\section{Methodology}

\section{Survey and data collection}

An ethnobotanical study was carried out between March 2017 and March 2019 to collect the knowledge of wild edible plant species being used by the local people in Yeșilli district and the surrounding areas. During this time, 120 plant specimens were collected. The collected plants were identified by the authors using 'Flora of Turkey' [13], 'A Checklist of the Flora of Turkey (Vascular Plants)' [16], 'Illustrated Flora of Turkey Vol 1' [17] and 'Illustrated Flora of Turkey Vol 2' [18]. Voucher specimens were deposited at the Herbarium of Faculty of Pharmacy, University of Istanbul (ISTE). The scientific names of the plant taxa were identified according to 'A Checklist of the Flora of Turkey (Vascular Plants)' [16] and The Plant List [104].

\section{Interviews with native people}

We conducted interviews with local people without much difficulty because two authors (B. Yılmaz and M. Çelik) 
are local to the area and have relations there. The local community was informed, and their permission was received before conducting the questionnaire. We received guidance such as from teachers, imams (ministers) of mosques or headmen of the villages. A questionnaire was administered to the local people through face-to-face interviews. Interviews were conducted in the fields, houses and common areas of the villages. We visited the fields during all seasons and visited all villages at least twice. Elderly people who possessed deep knowledge of plants were visited at least three times, and sometimes we spent 1 or 2 days with these people in their farms, gardens, natural areas or houses. The International Society of Ethnobiology Code of Ethics was taken into account in interviews [105].

\section{Data analysis}

The vegetable use category was further subcategorised as raw $\left(\mathrm{Veg}_{\mathrm{R}}\right)$, cooked $\left(\mathrm{Veg}_{\mathrm{C}}\right)$ or preserved $\left(\mathrm{Veg}_{\mathrm{P}}\right)$. Vegetables that are consumed after cooking, roasting or boiling, and those mixed with yogurt were categorised as cooked. Vegetables that are consumed after drying, adding to cheese or pickling were classified as preserved, and those consumed directly after washing, peeling or as salad were designated as raw.

Fruit usage was subcategorised as raw $\left(\mathrm{Fr}_{\mathrm{R}}\right)$ or processed $\left(\mathrm{Fr}_{\mathrm{P}}\right)$. Fruits that are eaten raw were categorised as raw, and processed fruits that are used after powdering, obtaining oil and drying were categorised as processed. Other categories included beverages (Bv), nectar (Ne), spices (Sp), latex (La) and medicinal plants (Med).

The information gathered through questionnaires and interviews was analysed quantitatively using two ethnobotanical indices as follows.

The factor informant consensus $\left(F_{i c}\right)$ was computed for each category to determine the homogeneity of the knowledge provided by the informants [105]. The Fic is calculated as follows:

$$
\text { Fic }=\frac{N_{u r}-N t}{N_{u r}-1}
$$

where $N_{\text {ur }}$ refers to the number of use reports from informants for a particular use category, and $N_{\mathrm{t}}$ refers to the number of taxa that are used for a particular use category by all informants.

The cultural importance (CI) index was calculated for each species using the following formula [105]:

CIs $=\left[\sum_{u^{\prime} \mathrm{u}_{1}}^{n_{\mathrm{N}}} \sum_{i=i_{1}}^{\mathrm{n}_{\mathrm{N}}} U R \frac{u i}{N}\right]$

The CI index can also be seen as the sum of the proportion of informants that mention the use of each species. This additive index takes into account not only the spread of the use (number of informants) for each species but also its versatility, i.e. the diversity of its uses.
The theoretical maximum value of the index is the total number of different use categories (NC), which is reached in the unlikely case that all informants mention the use of the species in all use categories considered in a survey [105].

Jaccard's similarity index considers the similarity between two OTUs (operational taxonomic units) as the number of attributes shared divided by the total number of attributes present in both OTUs. Jaccard's index may be expressed as follows

$$
\mathrm{J}=\mathrm{C} / \mathrm{A}+\mathrm{B}
$$

Where $A$ is the number of attributes present in OTU a, $B$ is the number of attributes present in OTU $\mathrm{b}$ and $C$ is the number of attributes present in both OTUs a and b. The number of attributes present in either of the OTUs is given by $A+B$ [106].

\section{Results and discussion}

Socio-economic characteristics of informants

A total of $62.9 \%$ female and $37.1 \%$ male informants were interviewed. The informants had varying levels of education, with $33.87 \%$ having no education, $46.77 \%$ having a primary level, $14.51 \%$ having a secondary level and only $4.83 \%$ having a tertiary level. The demographic details of the informants can be seen in Table 1.

\section{Diversity of wild edible plant taxa}

In multicultural regions, differences in the use of wild vegetables could be interpreted as a result of complex interactions between cultural preferences [99]. The use of plants varies in the present study area. The present study found 74 plant taxa belonging to 31 plant families that are used as wild edible plants in Yeșilli district (Mardin). Table 2 lists details of the utilised wild edible plant species arranged in alphabetical order, including scientific name, family name, herbarium number, local name (Arabic, A; Syriac, S; and Kurdish, K), life form, plant parts used, modes of consumption, $\mathrm{CI}$ and previous ethnobotanical literature records from

\begin{tabular}{lll}
\multicolumn{2}{l}{ Table 1 Demographic detail of the interviewed } & informants \\
\hline Category & Subcategory & \% of informants \\
\hline Gender & Female & 62.9 \\
& Male & 37.1 \\
Age & $15-40$ & 7 \\
& $40-60$ & 31 \\
& 60 and older & 24 \\
Education level & None & 33.87 \\
& Primary & 46.77 \\
& Secondary & 14.51 \\
& Tertiary & 4.83 \\
\hline
\end{tabular}


Table 2 List of wild plants used as foodstufs in Yeşilli District

\begin{tabular}{|c|c|c|c|c|c|c|c|c|}
\hline $\begin{array}{l}\text { Scientific name } \\
\text { Family name }\end{array}$ & $\begin{array}{l}\text { Voucher } \\
\text { number } \\
\text { (ISTE) }\end{array}$ & $\begin{array}{l}\text { Local name } \\
\text { (Languages; A, K, S) }\end{array}$ & $\begin{array}{l}\text { Ethnic } \\
\text { groups (Ar, } \\
\text { As, Ku) } \\
\text { (Number of } \\
\text { interviewes) }\end{array}$ & $\begin{array}{l}\text { Life } \\
\text { form }\end{array}$ & $\begin{array}{l}\text { Plant part } \\
\text { used }\end{array}$ & Modes of consumption & $\mathrm{Cl}$ & $\begin{array}{l}\text { Previous } \\
\text { ethnobotanical } \\
\text { literature records }\end{array}$ \\
\hline $\begin{array}{l}\text { Allium ampeloprasum } \mathrm{L} \text {. } \\
\text { Amarylidaceae }\end{array}$ & 115232 & $\begin{array}{l}\text { Summe }(A) \text {, } \\
\text { Sirik (K) }\end{array}$ & $\begin{array}{l}\text { As (1), Ku } \\
\text { (18) }\end{array}$ & Herb & $\begin{array}{l}\text { Leaves, } \\
\text { Bulbs }\end{array}$ & $\begin{array}{l}\text { Boiled then added to } \\
\text { yogurt and bulgur soup, } \\
\text { added into cheese } \\
\text { Raw as salad }\end{array}$ & 0.31 & {$[44,52,60,70,81]$} \\
\hline $\begin{array}{l}\text { Allium chloranthum } \\
\text { Boiss. } \\
\text { Amarylidaceae }\end{array}$ & 115738 & Serspik Sirik, Sirim (K) & $\mathrm{Ku}(10)$ & Herb & Leaves & $\begin{array}{l}\text { Added into cheese, raw, } \\
\text { added to salad }\end{array}$ & 0.16 & Not reported \\
\hline $\begin{array}{l}\text { Allium } \\
\text { wendelboanumKollmann }{ }^{\text {a }} \\
\text { Amarylidaceae }\end{array}$ & 115737 & Serspik, Sirik, Sirim (K) & Ku (6) & Herb & Leaves & $\begin{array}{l}\text { Added into cheese } \\
\text { Raw as salad }\end{array}$ & 0.09 & Not reported \\
\hline $\begin{array}{l}\text { Amaranthus retroflexus } \mathrm{L} \text {. } \\
\text { Amaranthaceae }\end{array}$ & 115734 & Koksor (K) & $\mathrm{Ku}(25)$ & Herb & $\begin{array}{l}\text { Aerial } \\
\text { parts }\end{array}$ & $\begin{array}{l}\text { Boiled then fried with egg, } \\
\text { cooked as soup with } \\
\text { yogurt and bulgur }\end{array}$ & 0.40 & $\begin{array}{l}{[32,33,37,40-42,} \\
50,51,69,80-82, \\
94]\end{array}$ \\
\hline $\begin{array}{l}\text { Anchusa strigosa Banks \& } \\
\text { Sol. } \\
\text { Boraginaceae }\end{array}$ & 115247 & Hımhım (A), Gûrîz (K) & $\begin{array}{l}\operatorname{Ar}(11), \mathrm{As} \\
(5), \mathrm{Ku}(39)\end{array}$ & Herb & $\begin{array}{l}\text { Young } \\
\text { leaves } \\
\text { Flowers } \\
\text { nectar }\end{array}$ & $\begin{array}{l}\text { Boiled then fried with } \\
\text { onion, medicinal } \\
\text { Its nectar sucked }\end{array}$ & 0.88 & {$[26,31,94]$} \\
\hline $\begin{array}{l}\text { Arum rupicola Boiss. } \\
\text { Araceae }\end{array}$ & 116014 & Kardi (K) & $\begin{array}{l}\operatorname{Ar}(3), \mathrm{Ku} \\
(10)\end{array}$ & Herb & Leaves & $\begin{array}{l}\text { Boiled then fried with } \\
\text { onion, medicinal }\end{array}$ & 0.21 & {$[55,70,72,94,98]$} \\
\hline $\begin{array}{l}\text { Capparis sicula subsp. } \\
\text { sicula Veill. } \\
\text { Capparaceae }\end{array}$ & 115267 & Kebere $(K)$ & $\begin{array}{l}\operatorname{Ar}(2), \mathrm{As} \\
(4), \mathrm{Ku}(14)\end{array}$ & Shrub & Buds & Raw as salad, pickled & 0.32 & $\begin{array}{l}{[26-28,41,52,} \\
56,65]\end{array}$ \\
\hline $\begin{array}{l}\text { Celtis tournefortii Lam. } \\
\text { Cannabaceae }\end{array}$ & 115293 & $\begin{array}{l}\text { Gernoso (S) } \\
\text { Gıngırêz (A) } \\
\text { Teêv, Tuêv (K) }\end{array}$ & $\begin{array}{l}\text { As (5), Ku } \\
(20)\end{array}$ & Tree & Fruits & $\begin{array}{l}\text { Raw as a snack, prepared a } \\
\text { mixture, medicinal }\end{array}$ & 0.40 & {$[28,40,49,81,82]$} \\
\hline $\begin{array}{l}\text { Centaurea hyalolepis } \\
\text { Boiss. } \\
\text { Asteraceae }\end{array}$ & 115257 & Sitrîzerk(K) & Ku (9) & Herb & $\begin{array}{l}\text { Young } \\
\text { leaves }\end{array}$ & Raw as salad & 0.14 & {$[26]$} \\
\hline $\begin{array}{l}\text { Centaurea virgata Lam. } \\
\text { Asteraceae }\end{array}$ & 115268 & Deqnişeh (A), Tal (K) & As (2) & Herb & $\begin{array}{l}\text { Young } \\
\text { leaves }\end{array}$ & Raw as salad & 0.03 & Not reported \\
\hline $\begin{array}{l}\text { Cerasus mahaleb var. } \\
\text { mahaleb (L.) Mill. } \\
\text { Rosaceae }\end{array}$ & 115684 & $\begin{array}{l}\text { Mahlab (S) } \\
\text { Mahlep, Mahleb } \\
(\text { A-K) }\end{array}$ & $\begin{array}{l}\text { As (5) } \\
\text { As (5), } \mathrm{Ku} \\
(4)\end{array}$ & Shrub & Fruits & $\begin{array}{l}\text { Prepared liqueur and wine } \\
\text { Raw as a snack, medicinal }\end{array}$ & $\begin{array}{l}0.08 \\
0.15\end{array}$ & [70] \\
\hline $\begin{array}{l}\text { Cerasus microcarpa } \\
\text { subsp. microcarpa } \\
\text { (C.A.Mey.) Boiss. } \\
\text { Rosaceae }\end{array}$ & 115677 & Fikt (A) & $\begin{array}{l}\text { As (3), Ku } \\
\text { (26) }\end{array}$ & Shrub & Fruits & Raw as a snack, medicinal & 0.29 & {$[49,60,94]$} \\
\hline $\begin{array}{l}\text { Cerasus prostrata var. } \\
\text { prostrata (Labill.) Ser. } \\
\text { Rosaceae }\end{array}$ & 115732 & $\begin{array}{l}\text { Belalûk (K) } \\
\text { Fıkt (A) } \\
\text { Horişmê (S) }\end{array}$ & $\mathrm{Ku}(11)$ & Shrub & Fruits & Raw as a snack, medicinal & 0.18 & Not reported \\
\hline $\begin{array}{l}\text { Chenopodium album } \mathrm{L} \text {. } \\
\text { Amaranthaceae }\end{array}$ & 115729 & Serbîmast (K) & $\mathrm{Ku}(12)$ & Herb & $\begin{array}{l}\text { Young } \\
\text { aerial } \\
\text { parts }\end{array}$ & $\begin{array}{l}\text { Boiled and then fried eggs } \\
\text { added, cooked a soup with } \\
\text { yogurt and bulgur }\end{array}$ & 0.19 & $\begin{array}{l}{[30,32-34,37,38,} \\
42,43,49,53,54, \\
59,63,65,69,71, \\
72,78-82,84,88, \\
89,92-94]\end{array}$ \\
\hline $\begin{array}{l}\text { Chondrilla juncea var. } \\
\text { juncea } \mathrm{L} \text {. } \\
\text { Asteraceae }\end{array}$ & 115248 & Ilke (A) & Ku (9) & Herb & $\begin{array}{l}\text { Young } \\
\text { leaves }\end{array}$ & $\begin{array}{l}\text { Raw as salad, added to } \\
\text { salad }\end{array}$ & 0.14 & $\begin{array}{l}{[33,38,41,42,50,} \\
65,78-80,94]\end{array}$ \\
\hline $\begin{array}{l}\text { Convolvulus arvensis L. } \\
\text { Convolvulaceae }\end{array}$ & 115674 & $\begin{array}{l}\text { Lavlavk (K), Lıblebê } \\
\text { (A) }\end{array}$ & $\begin{array}{l}\text { As (3), Ku } \\
\text { (16) }\end{array}$ & Herb & $\begin{array}{l}\text { Aerial } \\
\text { parts }\end{array}$ & $\begin{array}{l}\text { Boiled and then fried eggs } \\
\text { added, cooked a soup with } \\
\text { yogurt and bulgur }\end{array}$ & 0.19 & $\begin{array}{l}{[27,32,37,58,59,} \\
63,65,74,75,78, \\
89,92,94]\end{array}$ \\
\hline $\begin{array}{l}\text { Crataegus azarolus var. } \\
\text { azarolus } L \text {. } \\
\text { Rosaceae }\end{array}$ & 115250 & $\begin{array}{l}\text { Givîij, Gîjok, Gûhîi (K), } \\
\text { Ğıran (A) }\end{array}$ & $\begin{array}{l}\text { As (5), Ku } \\
\text { (33) }\end{array}$ & Tree & Fruits & Raw as a snack & 0.61 & {$[52,82,96]$} \\
\hline Crocus cancellatus & 115940 & Hılhılêye (A) & As (3), $\mathrm{Ku}$ & Herb & Corm & Raw as a snack after pelling & 0.61 & {$[26-28,79,94]$} \\
\hline
\end{tabular}


Table 2 List of wild plants used as foodstufs in Yeşilli District (Continued)

\begin{tabular}{|c|c|c|c|c|c|c|c|c|}
\hline $\begin{array}{l}\text { Scientific name } \\
\text { Family name }\end{array}$ & $\begin{array}{l}\text { Voucher } \\
\text { number } \\
\text { (ISTE) }\end{array}$ & $\begin{array}{l}\text { Local name } \\
\text { (Languages; A, K, S) }\end{array}$ & $\begin{array}{l}\text { Ethnic } \\
\text { groups (Ar, } \\
\text { As, Ku) } \\
\text { (Number of } \\
\text { interviewes) }\end{array}$ & $\begin{array}{l}\text { Life } \\
\text { form }\end{array}$ & $\begin{array}{l}\text { Plant part } \\
\text { used }\end{array}$ & Modes of consumption & $\mathrm{Cl}$ & $\begin{array}{l}\text { Previous } \\
\text { ethnobotanical } \\
\text { literature records }\end{array}$ \\
\hline $\begin{array}{l}\text { Herbert subsp. } \\
\text { damacenus (Herbert) } \\
\text { Mathew } \\
\text { Iridaceae }\end{array}$ & & Pîvok (K) & (35) & & & off the outher part & & \\
\hline $\begin{array}{l}\text { Echinophora tenuifolia L. } \\
\text { subsp. sibthorpiana } \\
\text { (Guss.) Tutin } \\
\text { Apiaceae }\end{array}$ & 115670 & Mırbela (A) & As (5) & Herb & $\begin{array}{l}\text { Young } \\
\text { stems }\end{array}$ & $\begin{array}{l}\text { Raw as a snack after } \\
\text { peeling off the outher part }\end{array}$ & 0.08 & $\begin{array}{l}{[26,27,41-43,49,} \\
58,74,78,81,92]\end{array}$ \\
\hline $\begin{array}{l}\text { Echinops orientalis Trautv. } \\
\text { Asteraceae }\end{array}$ & 115703 & $\begin{array}{l}\text { Gelo helvo pevero } \\
\text { (S) }\end{array}$ & $\begin{array}{l}\text { As (5) } \\
\mathrm{Ku}(21)\end{array}$ & Herb & $\begin{array}{l}\text { Receptacle } \\
\text { Dried } \\
\text { latex }\end{array}$ & Raw as a snack & 0.16 & {$[27,40,60,70,78]$} \\
\hline $\begin{array}{l}\text { Echinops spinosissimus } \\
\text { Turra subsp. bithynicus } \\
\text { (Boiss.) Greuter } \\
\text { Asteraceae }\end{array}$ & 115283 & $\begin{array}{l}\text { Gelo helvo pevero } \\
\text { (S) }\end{array}$ & As (5) & Herb & $\begin{array}{l}\text { Receptacle } \\
\text { Dried } \\
\text { latex }\end{array}$ & Raw as a snack & 0.08 & [40] \\
\hline $\begin{array}{l}\text { Echium italicum L. } \\
\text { Boraginaceeae }\end{array}$ & 115234 & Hımhım (A), Mijok (K) & $\begin{array}{l}\text { As }(2), \mathrm{Ku} \\
\text { (9) }\end{array}$ & Herb & $\begin{array}{l}\text { Flowers, } \\
\text { nectar }\end{array}$ & Its nectar sucked, medicinal & 0.18 & {$[26,40-42]$} \\
\hline $\begin{array}{l}\text { Elaeagnus angustifolia L. } \\
\text { Elaeagnaceae }\end{array}$ & 115235 & $\operatorname{Sinç}(K)$ & $\mathrm{Ku}(31)$ & Shrub & $\begin{array}{l}\text { Fruits } \\
\text { Flowers }\end{array}$ & $\begin{array}{l}\text { Raw as a snack } \\
\text { As tea }\end{array}$ & 0.50 & $\begin{array}{l}{[32,36,37,49,52} \\
69,78,79,93,94]\end{array}$ \\
\hline $\begin{array}{l}\text { Erodium cicutarium (L.) } \\
\text { L'Hér } \\
\text { Geraniaceae }\end{array}$ & 116438 & Derzikepire (K) & $\begin{array}{l}\operatorname{Ar}(5), \mathrm{Ku} \\
(15)\end{array}$ & Herb & $\begin{array}{l}\text { Leaves, } \\
\text { fruits, } \\
\text { stems }\end{array}$ & $\begin{array}{l}\text { Eaten raw } \\
\text { Raw as a snack after pelling } \\
\text { off the outher part }\end{array}$ & 0.32 & $\begin{array}{l}{[26,31,41,42,50,} \\
57,58,65,73,75, \\
89,99]\end{array}$ \\
\hline $\begin{array}{l}\text { Eryngium creticum Lam. } \\
\text { Apiaceae }\end{array}$ & 115236 & Beektire/Ekkeyde (A) & $\begin{array}{l}\operatorname{Ar}(15), \text { As } \\
(4)\end{array}$ & Herb & $\begin{array}{l}\text { Petioles } \\
\text { Young } \\
\text { stem, } \\
\text { young } \\
\text { roots }\end{array}$ & $\begin{array}{l}\text { Raw as a snack } \\
\text { Raw as a snack after pelling } \\
\text { off the outher part, } \\
\text { medicinal }\end{array}$ & 0.29 & {$[48,49,73]$} \\
\hline $\begin{array}{l}\text { Euphorbia helioscopia } \\
\text { subsp. helioscopia L. } \\
\text { Euphorbiaceae }\end{array}$ & 115254 & Lahye (A) & $\begin{array}{l}\text { As }(2), \mathrm{Ku} \\
\text { (8) }\end{array}$ & Herb & $\begin{array}{l}\text { Aerial } \\
\text { parts }\end{array}$ & $\begin{array}{l}\text { Added to molasses, to } \\
\text { improve the test of } \\
\text { molasses, direct or dry }\end{array}$ & 0.16 & [41] \\
\hline $\begin{array}{l}{ }^{b} \text { Ficus carica subsp. } \\
\text { carica L. } \\
\text { Moraceae }\end{array}$ & 115943 & $\begin{array}{l}\text { Hejîr (K) } \\
\text { Tin (A) }\end{array}$ & $\begin{array}{l}\operatorname{Ar}(15), \mathrm{As} \\
(5), \mathrm{Ku}(39)\end{array}$ & Tree & Fruits & Raw as a snack, dried eaten & 0.96 & $\begin{array}{l}{[20,26,37,38,40} \\
43,50,52,55,56 \\
58,59,62,63,72 \\
75,82,86,92,96]\end{array}$ \\
\hline Ficus carica L. subsp. & 115699 & Kerik hejîr (K), Tin (A) & As (5), $\mathrm{Ku}$ & Shrub & Fruits & Raw as a snack, dried eaten & 0.32 & {$[27,40,94]$} \\
\hline
\end{tabular}

rupestris (Hausskn.)

(15)

Moraceae

\section{Gagea villosa (M.Bieb.) \\ Sweet \\ Liliaceae}

Geocaryum cynapioides (Guss.) Engstrand subsp. macrocarpum (Boiss. \&

Spruner) Menemen

Apiaceae

Glycyrrhiza glabra L. Fabaceae

Gundelia tournefortii L. Asteraceae
116418

115681 Ceviz il arz (A) Gûzê ardê (K)

115667 $M$
Ar (12), As Herb Roots

115711 Arkue (A),

Ergudê (S)

Kereng, Herşev (K),
(5), Ku (34)

Ar (6), As Herb Petioles

(5), $\mathrm{Ku}$ (39) Roots,
Prepared as syrup, medicinal

Raw as a snack Young stems and roots are eaten after pelling off the outher part as a snack, boiled and then fried, eggs added, boiled and then the soup is preparedby adding
0.16 Not reported

0.08 Not reported
$0.83[26,27,32,41,48$, $52,78,79,81,82$, 93]

$0.80 \quad[26,27,31,33,40-$ $42,52,55,60,65$, $70,72,78,80-82$, $84,86,89,94,96$, $98,99]$ 
Table 2 List of wild plants used as foodstufs in Yeşilli District (Continued)

\begin{tabular}{|c|c|c|c|c|c|c|c|c|}
\hline $\begin{array}{l}\text { Scientific name } \\
\text { Family name }\end{array}$ & $\begin{array}{l}\text { Voucher } \\
\text { number } \\
\text { (ISTE) }\end{array}$ & $\begin{array}{l}\text { Local name } \\
\text { (Languages; A, K, S) }\end{array}$ & $\begin{array}{l}\text { Ethnic } \\
\text { groups (Ar, } \\
\text { As, Ku) } \\
\text { (Number of } \\
\text { interviewes) }\end{array}$ & $\begin{array}{l}\text { Life } \\
\text { form }\end{array}$ & $\begin{array}{l}\text { Plant part } \\
\text { used }\end{array}$ & Modes of consumption & $\mathrm{Cl}$ & $\begin{array}{l}\text { Previous } \\
\text { ethnobotanical } \\
\text { literature records }\end{array}$ \\
\hline & & & & & & yogurt and bulgur & & \\
\hline $\begin{array}{l}\text { Hirschfeldia incana (L.) } \\
\text { Lagr.-Foss. } \\
\text { Brassicaceae }\end{array}$ & 115307 & $\begin{array}{l}\text { Herdlo (S) } \\
\text { Xerdel (A-K) }\end{array}$ & $\mathrm{Ku}(7)$ & Herb & $\begin{array}{l}\text { Young } \\
\text { aerial } \\
\text { parts }\end{array}$ & $\begin{array}{l}\text { Raw as a snack, as salad, } \\
\text { medicinal }\end{array}$ & 0.11 & [107] \\
\hline $\begin{array}{l}\text { Iris persica } \mathrm{L} \text {. } \\
\text { Iridaceae }\end{array}$ & 116013 & $\begin{array}{l}\text { Birbîzêk, } \\
\text { Birbîzêka mîha (K), } \\
\text { Birxızeylê }(A)\end{array}$ & $\begin{array}{l}\text { As (2) } \\
\mathrm{Ku}(8)\end{array}$ & Herb & Tepals & Raw as a snack & 0.16 & {$[82,94]$} \\
\hline $\begin{array}{l}\text { Iris reticulata M. Bieb. } \\
\text { Iridaceae }\end{array}$ & 116000 & $\begin{array}{l}\text { Birbîzêk, } \\
\text { Birbîzêka bizina (K), } \\
\text { Birxızeylê (A) }\end{array}$ & $\begin{array}{l}\text { As (2), } \\
\mathrm{Ku}(8)\end{array}$ & Herb & Tepals & Raw as a snack & 0.16 & {$[31,82]$} \\
\hline $\begin{array}{l}\text { bJuglans regia } \mathrm{L} \text {. } \\
\text { Juglandaceae }\end{array}$ & 115679 & $\begin{array}{l}\text { Cevz (A) } \\
\text { Gûz (K) }\end{array}$ & $\begin{array}{l}\operatorname{Ar}(12), \mathrm{As} \\
(5), \mathrm{Ku}(28)\end{array}$ & Tree & Seeds & Eaten as dried nuts & 0.73 & $\begin{array}{l}{[20,28,35-38,40,} \\
41,49,55,57,60, \\
62,64,66,68,70- \\
72,82,92,94]\end{array}$ \\
\hline $\begin{array}{l}\text { Lathyrus cassius Boiss. } \\
\text { Fabaceae }\end{array}$ & 115721 & Baqilê bizinan (K) & $\mathrm{Ku}(10)$ & Herb & $\begin{array}{l}\text { Young } \\
\text { seeds }\end{array}$ & Raw as a snack & 0.16 & Not reported \\
\hline $\begin{array}{l}\text { Lepidium draba L. } \\
\text { Brassicaceae }\end{array}$ & 115252 & Qınêber (K) & $\begin{array}{l}\operatorname{Ar}(18), \mathrm{As} \\
(5), \mathrm{Ku}(37)\end{array}$ & Herb & $\begin{array}{l}\text { Young } \\
\text { aerial } \\
\text { parts }\end{array}$ & $\begin{array}{l}\text { Raw as a snack, as salad, } \\
\text { fried with salad and eggs } \\
\text { added }\end{array}$ & 0.96 & $\begin{array}{l}{[26,27,31,32,42,} \\
44,53,55,69,81, \\
84,89,93]\end{array}$ \\
\hline $\begin{array}{l}\text { Malva neglecta Wallr. } \\
\text { Malvaceae }\end{array}$ & 115242 & $\begin{array}{l}\text { Hıbbeze (A), Tolig, } \\
\text { Tolik (K) }\end{array}$ & $\begin{array}{l}\operatorname{Ar}(10), \mathrm{As} \\
(4), \mathrm{Ku}(34) \\
\mathrm{Ku}(30)\end{array}$ & Herb & $\begin{array}{l}\text { Young } \\
\text { aerial } \\
\text { parts } \\
\text { Fruit }\end{array}$ & $\begin{array}{l}\text { Boiled and then fried with } \\
\text { onion, medicinal } \\
\text { Raw as a snack }\end{array}$ & 0.77 & $\begin{array}{l}{[26,28,30-34,36,} \\
38,40,44,47-49, \\
51-55,59,62,65, \\
76,78-82,84,88, \\
89,94,98,99]\end{array}$ \\
\hline $\begin{array}{l}\text { Mentha longifolia (L.) L. } \\
\text { Lamiaceae }\end{array}$ & 115243 & $\begin{array}{l}\text { Nahne, Ninha (A), } \\
\text { Pûng (K) }\end{array}$ & $\begin{array}{l}\operatorname{Ar}(18), \mathrm{As} \\
(3), \mathrm{Ku}(26)\end{array}$ & Herb & $\begin{array}{l}\text { Leaves, } \\
\text { young } \\
\text { aerial } \\
\text { parts }\end{array}$ & $\begin{array}{l}\text { As spices, medicinal } \\
\text { As tea }\end{array}$ & 0.75 & $\begin{array}{l}{[32-34,38,40,44,} \\
47-49,51-54,58, \\
60,62,63,65,69, \\
70,72,74-76,78, \\
80-82,84-89,94, \\
96,98,99]\end{array}$ \\
\hline $\begin{array}{l}\text { Nasturtium officinale R.Br., } \\
\text { Aiton } \\
\text { Brassicaceae }\end{array}$ & 115903 & Tûzik (K) & $\mathrm{Ku}(31)$ & Herb & $\begin{array}{l}\text { Young } \\
\text { aerial part }\end{array}$ & Raw as a snack, as salad & 0.50 & $\begin{array}{l}{[41-43,51,55,59,} \\
64,71,74,76,78, \\
80,82,98,99]\end{array}$ \\
\hline $\begin{array}{l}\text { Notobasis syriaca (L.) } \\
\text { Cass. } \\
\text { Asteraceae }\end{array}$ & 116415 & $\begin{array}{l}\text { Aynbeloqê (A) } \\
\text { Kelbeşe belek (K) }\end{array}$ & $\begin{array}{l}\operatorname{Ar}(7), A s \\
(4), K u(39)\end{array}$ & Herb & $\begin{array}{l}\text { Young } \\
\text { stems }\end{array}$ & $\begin{array}{l}\text { Raw as a snack after pelling } \\
\text { off the outher part }\end{array}$ & 0.80 & {$[26,28,50,96]$} \\
\hline $\begin{array}{l}{ }^{\mathrm{b}} \text { Olea europaea L. } \\
\text { Oleaceae }\end{array}$ & 115262 & Zeitûn (A-K) & $\begin{array}{l}\operatorname{Ar}(8), \mathrm{As} \\
(5), \mathrm{Ku}(20)\end{array}$ & Tree & Fruit, oil & Eaten conserved, medicinal & 0.50 & $\begin{array}{l}{[37,38,42,50,71,} \\
92]\end{array}$ \\
\hline $\begin{array}{l}\text { Onopordum } \\
\text { carduchorum Bornm. \& } \\
\text { Beauverd } \\
\text { Asteraceae }\end{array}$ & 116442 & Kelbeşakaran (K) & $\begin{array}{l}\operatorname{Ar}(6), A s \\
(5), K u(39)\end{array}$ & Herb & $\begin{array}{l}\text { Young } \\
\text { stems }\end{array}$ & $\begin{array}{l}\text { Raw as a snack after pelling } \\
\text { off the outher part }\end{array}$ & 0.80 & {$[28,31,55]$} \\
\hline $\begin{array}{l}\text { Onosma roussaei DC. } \\
\text { Boraginaceae }\end{array}$ & 115712 & Hımhım (A), Mîjok (K) & $\mathrm{Ku}(7)$ & Herb & $\begin{array}{l}\text { Flowers } \\
\text { nectar }\end{array}$ & Its nectar sucked & 0.12 & [31] \\
\hline $\begin{array}{l}\text { Onosma alborosea Fisch. } \\
\text { \& C.A.Mey. } \\
\text { Boraginaceae }\end{array}$ & 115316 & $\begin{array}{l}\text { Hımhım }(A) \text {, } \\
\text { Mîjok (K) }\end{array}$ & $\begin{array}{l}\text { As (2), Ku } \\
(10)\end{array}$ & Herb & $\begin{array}{l}\text { Flowers } \\
\text { nectar }\end{array}$ & Its nectar sucked & 0.19 & Not reported \\
\hline $\begin{array}{l}\text { Papaver glaucum Boiss. \& } \\
\text { Hausskn. Ex Boiss. } \\
\text { Papaveraceae }\end{array}$ & 115249 & $\begin{array}{l}\text { Kulîlka Bûk û Zava, } \\
\text { Kulîlka Nisanê, Kulîlka } \\
\text { erebo (K), Qırçeh (A) }\end{array}$ & $\mathrm{Ku}(12)$ & Herb & $\begin{array}{l}\text { Young } \\
\text { leaves }\end{array}$ & $\begin{array}{l}\text { Raw as salad, fried, } \\
\text { medicinal }\end{array}$ & 0.19 & Not reported \\
\hline $\begin{array}{l}\text { Papaver macrostomum } \\
\text { Boiss. \& A.Huet } \\
\text { Papaveraceae }\end{array}$ & 115680 & Kulîlka Nisanê (K) & $\begin{array}{l}\text { As (3), Ku } \\
(32)\end{array}$ & Herb & $\begin{array}{l}\text { Young } \\
\text { leaves }\end{array}$ & $\begin{array}{l}\text { Raw as salad, fried, } \\
\text { medicinal. }\end{array}$ & 0.22 & {$[65,78,94,99]$} \\
\hline $\begin{array}{l}\text { Pistacia terebinthus } \mathrm{L} \text {. } \\
\text { subsp. palaestina (Boiss.) }\end{array}$ & 115944 & $\begin{array}{l}\text { Batmê (S) } \\
\text { Bittim (K) }\end{array}$ & $\begin{array}{l}\operatorname{Ar}(10), \text { As } \\
(4), \mathrm{Ku}(16)\end{array}$ & Shrub & $\begin{array}{l}\text { Unripe } \\
\text { fruits }\end{array}$ & $\begin{array}{l}\text { Raw as a snack or } \\
\text { preparing a coffee }\end{array}$ & 0.48 & $\begin{array}{l}{[30,38,41-43,50,} \\
52,56,59,60,65,\end{array}$ \\
\hline
\end{tabular}


Table 2 List of wild plants used as foodstufs in Yeşilli District (Continued)

\begin{tabular}{|c|c|c|c|c|c|c|c|c|}
\hline $\begin{array}{l}\text { Scientific name } \\
\text { Family name }\end{array}$ & $\begin{array}{l}\text { Voucher } \\
\text { number } \\
\text { (ISTE) }\end{array}$ & $\begin{array}{l}\text { Local name } \\
\text { (Languages; A, K, S) }\end{array}$ & $\begin{array}{l}\text { Ethnic } \\
\text { groups (Ar, } \\
\text { As, Ku) } \\
\text { (Number of } \\
\text { interviewes) }\end{array}$ & $\begin{array}{l}\text { Life } \\
\text { form }\end{array}$ & $\begin{array}{l}\text { Plant part } \\
\text { used }\end{array}$ & Modes of consumption & $\mathrm{Cl}$ & $\begin{array}{l}\text { Previous } \\
\text { ethnobotanical } \\
\text { literature records }\end{array}$ \\
\hline $\begin{array}{l}\text { Engler } \\
\text { Anacardiaceae }\end{array}$ & & Bıttım (A) & & & & & & $75,78,81,86]$ \\
\hline $\begin{array}{l}\text { Pisum fulvum Sibth. \& } \\
\text { Sm. } \\
\text { Fabaceae }\end{array}$ & 115724 & Baqilê xatûnî (K) & $\mathrm{Ku}(10)$ & Herb & $\begin{array}{l}\text { Young } \\
\text { seeds }\end{array}$ & Raw as a snack & 0.16 & Not reported \\
\hline $\begin{array}{l}\text { Polygonum cognatum } \\
\text { Meissn. } \\
\text { Polygonaceae }\end{array}$ & 115275 & Casûrik (K) & $\operatorname{Ar}(5)$ & Herb & $\begin{array}{l}\text { Young } \\
\text { aerial } \\
\text { parts }\end{array}$ & $\begin{array}{l}\text { Raw as a snack, as salad, } \\
\text { fried or boiled then added } \\
\text { to yogurt }\end{array}$ & 0.08 & $\begin{array}{l}{[32-36,40-44,48,} \\
49,52,54,55,58, \\
59,65,70,75,76, \\
78,80,81,84,88, \\
89,93,94]\end{array}$ \\
\hline $\begin{array}{l}\text { Portulaca oleracea L. } \\
\text { Portulacaceae }\end{array}$ & 115241 & $\begin{array}{l}\text { Pirpar (A-K), Parparık } \\
(\mathrm{K})\end{array}$ & $\begin{array}{l}\text { As (5), Ku } \\
\text { (19) }\end{array}$ & Herb & $\begin{array}{l}\text { Aerial } \\
\text { parts }\end{array}$ & $\begin{array}{l}\text { Raw in salads, cooked as } \\
\text { vegetable, medicinal. }\end{array}$ & 0.38 & $\begin{array}{l}{[31,33,55,59,70} \\
75,82,96,98]\end{array}$ \\
\hline $\begin{array}{l}\text { Quercus brantii Lindl. } \\
\text { Fagaceae }\end{array}$ & 115902 & Berû $(K)$ & $\begin{array}{l}\text { As }(1), \mathrm{Ku} \\
(17)\end{array}$ & Tree & Seed & $\begin{array}{l}\text { Roasted in embers, } \\
\text { medicinal }\end{array}$ & 0.29 & {$[31,38,52]$} \\
\hline $\begin{array}{l}\text { Rosa canina L. } \\
\text { Rosaceae }\end{array}$ & 115255 & Gulşîlav (K) & $\begin{array}{l}\text { As (5), Ku } \\
\text { (28) }\end{array}$ & Shrub & Fruits & Raw as a snack, medicinal & 0.53 & $\begin{array}{l}{[20,32-36,38,40,} \\
41,47-49,51-53, \\
55,59,60,62,63, \\
65,66,69-72,74, \\
75,78,81,82,86, \\
93,94,96]\end{array}$ \\
\hline $\begin{array}{l}\text { Rosa foetida J. Herrm. } \\
\text { Rosaceae }\end{array}$ & 116444 & $\begin{array}{l}\text { Verdasfar (A), } \\
\text { Gulşîlave zer (K) }\end{array}$ & $\begin{array}{l}\text { As (4), Ku } \\
\text { (36) }\end{array}$ & Shrub & Petals & Raw as a snack & 0.65 & Not reported \\
\hline $\begin{array}{l}\text { Rosularia radiciflora } \\
\text { Borris. } \\
\text { Crassulaceae }\end{array}$ & 116459 & $\begin{array}{l}\text { Tirîke pîrê (K) } \\
\text { Tulfilasfor (A) }\end{array}$ & $\begin{array}{l}\text { As }(2), \mathrm{Ku} \\
(5)\end{array}$ & Herb & Leaves & Raw as a snack & 0.11 & Not reported \\
\hline $\begin{array}{l}\text { Rhus coriaria } \mathrm{L} \text {. } \\
\text { Anacardiaceae }\end{array}$ & 115280 & Simaq $(A-K)$ & $\begin{array}{l}\operatorname{Ar}(18), \text { As } \\
\text { (5), Ku (32) }\end{array}$ & Shrub & Fruit & As spice & 0.88 & $\begin{array}{l}{[20,28,31,34,38,} \\
41,52,55,60,65 \\
66,71,75,78,81 \\
82,86,92,96]\end{array}$ \\
\hline $\begin{array}{l}\text { Rubus sanctus Schreb. } \\
\text { Rosaceae }\end{array}$ & 115306 & $\begin{array}{l}\text { Durîreşk, Durîhişhişok } \\
\text { (K) }\end{array}$ & $\begin{array}{l}\text { As (3), Ku } \\
(30)\end{array}$ & Shrub & Fruits & Raw as a snack & 0.54 & $\begin{array}{l}{[20,30,31,37,38,} \\
49,50,55,60,63, \\
66,71,75,81,82, \\
92]\end{array}$ \\
\hline $\begin{array}{l}\text { Rumex crispus L. } \\
\text { Polygonaceae }\end{array}$ & 115271 & $\begin{array}{l}\text { Sumaksevaqi (A), } \\
\text { Tirşuq (K) }\end{array}$ & $\begin{array}{l}\text { As (3), Ku } \\
\text { (9) }\end{array}$ & Herb & $\begin{array}{l}\text { Young } \\
\text { leaves }\end{array}$ & $\begin{array}{l}\text { As a wrapping material for } \\
\text { 'sarma' }\end{array}$ & 0.19 & $\begin{array}{l}{[32,33,36,37,41-} \\
43,49,52-55,58, \\
65,69,71,75,76, \\
78,80,84,88,96, \\
98]\end{array}$ \\
\hline $\begin{array}{l}\text { Rumex obtusifolius L. } \\
\text { subps. subalpinus (Schur) } \\
\text { Celak. } \\
\text { Polygonaceae }\end{array}$ & 115309 & $\begin{array}{l}\text { Sumaksevaqi }(A), \\
\text { Sabûna leglegê (K) }\end{array}$ & $\begin{array}{l}\text { As (3), Ku } \\
\text { (12) }\end{array}$ & Herb & $\begin{array}{l}\text { Young } \\
\text { leaves }\end{array}$ & $\begin{array}{l}\text { As a wrapping material for } \\
\text { 'sarma' }\end{array}$ & 0.24 & {$[41,45,75]$} \\
\hline $\begin{array}{l}\text { Rumex tuberosus L. } \\
\text { subsp. horizantalis (Koch) } \\
\text { Rech. } \\
\text { Polygonaceae }\end{array}$ & 115256 & $\begin{array}{l}\text { Sumaksevaqi (A), } \\
\text { Tirşuq (K) }\end{array}$ & $\begin{array}{l}\text { As }(4), \mathrm{Ku} \\
(7)\end{array}$ & Herb & $\begin{array}{l}\text { Young } \\
\text { aerial } \\
\text { parts }\end{array}$ & Raw as a snack & 0.18 & $\begin{array}{l}{[30,32,36,44,47,} \\
53,59,60,70,72, \\
76,94]\end{array}$ \\
\hline $\begin{array}{l}\text { Salvia syriaca L. } \\
\text { Lamiaceae }\end{array}$ & 115671 & Carboe (A) & $\operatorname{Ar}(14)$ & Herb & Gall & Raw as a snack & 0.23 & {$[31,52,94]$} \\
\hline $\begin{array}{l}\text { Sedum pallidum Bieb. } \\
\text { Crassulaceae }\end{array}$ & 116434 & $\begin{array}{l}\text { Tirîke pîrê (K) } \\
\text { Tulfilasfor (A) }\end{array}$ & $\begin{array}{l}\text { As }(2), \mathrm{Ku} \\
(5)\end{array}$ & Herb & Leaves & Raw as a snack & 0.11 & Not reported \\
\hline $\begin{array}{l}\text { Silene dichotoma Erh. } \\
\text { Caryophyllaceae }\end{array}$ & 116417 & Şekirok (K) & As (5) & Herb & $\begin{array}{l}\text { Young } \\
\text { seeds }\end{array}$ & Raw as a snack & 0.08 & {$[31,33,55]$} \\
\hline $\begin{array}{l}\text { Sinapis alba subsp. alba } \\
\text { L. } \\
\text { Brassicaceae }\end{array}$ & 115315 & Xerdel (A-K) & $\begin{array}{l}\operatorname{Ar}(18), \text { As } \\
(4), \mathrm{Ku}(29)\end{array}$ & Herb & $\begin{array}{l}\text { Young } \\
\text { aerial } \\
\text { parts }\end{array}$ & $\begin{array}{l}\text { Fried with onion } \\
\text { Raw as a snack }\end{array}$ & 0.82 & $\begin{array}{l}{[26,28,41,42,92,} \\
99]\end{array}$ \\
\hline
\end{tabular}


Table 2 List of wild plants used as foodstufs in Yeşilli District (Continued)

\begin{tabular}{|c|c|c|c|c|c|c|c|c|}
\hline $\begin{array}{l}\text { Scientific name } \\
\text { Family name }\end{array}$ & $\begin{array}{l}\text { Voucher } \\
\text { number } \\
\text { (ISTE) }\end{array}$ & $\begin{array}{l}\text { Local name } \\
\text { (Languages; A, K, S) }\end{array}$ & $\begin{array}{l}\text { Ethnic } \\
\text { groups (Ar, } \\
\text { As, Ku) } \\
\text { (Number of } \\
\text { interviewes) }\end{array}$ & $\begin{array}{l}\text { Life } \\
\text { form }\end{array}$ & $\begin{array}{l}\text { Plant part } \\
\text { used }\end{array}$ & Modes of consumption & $\mathrm{Cl}$ & $\begin{array}{l}\text { Previous } \\
\text { ethnobotanical } \\
\text { literature records }\end{array}$ \\
\hline & & & & & $\begin{array}{l}\text { Young } \\
\text { leaves }\end{array}$ & & & \\
\hline $\begin{array}{l}\text { Thymbra spicata subsp. } \\
\text { spicata L. } \\
\text { Lamiaceae }\end{array}$ & 115700 & Cehter, Cahter (K) & $\begin{array}{l}\text { As }(4), \mathrm{Ku} \\
(25)\end{array}$ & Shrub & $\begin{array}{l}\text { Aerial } \\
\text { parts }\end{array}$ & As spice & 0.46 & $\begin{array}{l}{[28,30,31,38,43,} \\
55,74,78,86]\end{array}$ \\
\hline $\begin{array}{l}\text { Thymus kotschyanus } \\
\text { subsp. kotschyanus Boiss. } \\
\text { \& Hohen. } \\
\text { Lamiaceae }\end{array}$ & 115706 & Cehter, Cahter (K) & Ku (9) & Shrub & $\begin{array}{l}\text { Aerial } \\
\text { parts }\end{array}$ & As spice & 0.14 & $\begin{array}{l}{[32,38,60,62,70,} \\
74,78,81,82]\end{array}$ \\
\hline $\begin{array}{l}\text { Tragopogon latifolius } \\
\text { Boiss. var. angustifolius } \\
\text { Boiss. } \\
\text { Asteraceae }\end{array}$ & 115687 & Gezrik (K) & $\mathrm{Ku}(7)$ & Herb & $\begin{array}{l}\text { Young } \\
\text { leaves and } \\
\text { roots }\end{array}$ & Raw as a snack & 0.11 & $\begin{array}{l}{[33,42,58,65,89,} \\
94]\end{array}$ \\
\hline $\begin{array}{l}\text { Tragopogon porrifolius L. } \\
\text { subsp. longirostris (Sch. } \\
\text { Bip.) Greuter } \\
\text { Asteraceae }\end{array}$ & 116428 & Gezrik (K) & Ku (6) & Herb & $\begin{array}{l}\text { Young } \\
\text { leaves and } \\
\text { roots }\end{array}$ & Raw as a snack & 0.10 & {$[31,41,42,99]$} \\
\hline $\begin{array}{l}\text { Urtica dioica } \mathrm{L} . \\
\text { Urticaceae }\end{array}$ & 115253 & $\begin{array}{l}\text { Gezgezik (K) } \\
\text { Kırays (A) }\end{array}$ & $\begin{array}{l}\operatorname{Ar}(14), \text { As } \\
\text { (5), Ku (26) }\end{array}$ & Herb & $\begin{array}{l}\text { Young } \\
\text { aerial } \\
\text { parts }\end{array}$ & $\begin{array}{l}\text { Fried with onion and then } \\
\text { eggs added, medicinal }\end{array}$ & 0.72 & $\begin{array}{l}{[20,26,28,30-34,} \\
36,40,42-44,48- \\
50,52-55,58-60, \\
62-65,69-72,75- \\
81,86,88,89,92, \\
94,98]\end{array}$ \\
\hline $\begin{array}{l}\text { Urtica pilulifera L. } \\
\text { Urticaceae }\end{array}$ & 115258 & Gezgezok (K) & $\mathrm{Ku}(15)$ & Herb & $\begin{array}{l}\text { Young } \\
\text { aerial } \\
\text { parts }\end{array}$ & $\begin{array}{l}\text { Raw as a snack, fried with } \\
\text { onion and then eggs } \\
\text { added, medicinal }\end{array}$ & 0.24 & {$[30,33,36,55]$} \\
\hline $\begin{array}{l}\text { Vicia palaestina Boiss. } \\
\text { Fabaceae }\end{array}$ & 115723 & Baqilê çukan (K) & $\mathrm{Ku}(11)$ & Herb & $\begin{array}{l}\text { Young } \\
\text { seed }\end{array}$ & Raw as a snack & 0.18 & {$[26]$} \\
\hline $\begin{array}{l}\text { Vicia pannonica var. } \\
\text { pannonica Crantz } \\
\text { Fabaceae }\end{array}$ & 115716 & Baqilê gan (K) & $\mathrm{Ku}(10)$ & Herb & $\begin{array}{l}\text { Young } \\
\text { seed }\end{array}$ & Raw as a snack & 0.16 & [31] \\
\hline $\begin{array}{l}\text { Vicia sativa L. subsp. } \\
\text { nigra (L.) Ehrh. var. nigra } \\
\text { Fabaceae }\end{array}$ & 115722 & Baqilê maran (K) & $\mathrm{Ku}(18)$ & Herb & $\begin{array}{l}\text { Young } \\
\text { seed }\end{array}$ & Raw as a snack & 0.29 & {$[27,49,52,63]$} \\
\hline
\end{tabular}

ISTE Herbarium of Faculty of Pharmacy, University of Istanbul, A Arabic, Ar Arab, As Assyrian, K Kurdish, Ku Kurd, S Syriac ${ }^{\mathrm{a} E n d e m i c}$

${ }^{\mathrm{b}}$ Both cultivated and wild plants

Turkey and bordering countries. The results show that most of the plants used are Asteraceae (ten taxa), followed by Rosaceae (seven taxa), Fabaceae (six taxa), Lamiaceae (four taxa), Brassicaceae (four taxa), Polygonaceae (four taxa) and Boraginaceae (three taxa). The remaining plant families are represented by only one or two species. Because of the dominance of the Asteraceae, Rosaceae and Fabaceae families in the flora of the studied area, plants from these families are used extensively. These results are in general agreement with those of previous studies [2, 29, 40, 81, 99]. However, some of previous studies $[60,70,82]$ reported that most of the wild edible plants were in the Apiaceae family. While others [68] reported that Fabaceae is the most important family of wild edible plants. The use of plants from different families indicates the intensity and importance of this information. These results are in accordance with those found in studies conducted in Turkey [31, 32, 60, 70, 81, 82] and bordering countries [97-99]. However, in some studies conducted in other parts of the world, the most commonly used plants are in Rosaceae family [1-4, 108, 109].

When comparing the results of the present study with those of ethnobotanical studies in Turkey, the regions in which plants and their use most overlap are as follows, listed according to the number of taxa: Midyat (Mardin) (23 taxa) [31], Malatya (22 taxa) [94], Bingöl (19 taxa) [82], Cizre (Şırnak) (18 taxa) [52], Elazığ (17 taxa) [81], Urfa (16 taxa) [26], Tunceli (15 taxa) [40] and Hakkari (13 taxa) [60]. Since the region where this present study was conducted is close to Midyat [31] and has the same cultural structure and geography as Mardin, some of the plants and 
their uses overlap with those identified in the Midyat study, as were expected. Other areas where overlaps are intense are Turkey's southeast (including Yeșilli), and the Eastern Anatolia Region. These regions are located in the same phytogeographic region and have almost the same cultural structure. Also, overlaps were observed with ethnobotanical studies conducted in bordering countries with the same cultural structure: Iraq (11 taxa) [99], southern Iraq (9 taxa) [96], Hawraman (Iraq) (8 taxa) [98] and Armenia (8 taxa) [97].

Additionally, when our results were compared with those of an ethnobotanical study conducted in Midyat, close to Yeşilli, we observed many similarities between the local names of plants because of the similar cultural structures areas. The local names of 14 taxa are similar: Cerasus mahaleb var. mahaleb, Convolvulus arvensis, Erodium cicutarium, Gundelia tourneforti, Malva neglecta, Pistacia terebinthus subp. palaestina, Rhus coriaria, Thymbra spicata and Urtica dioica. Also, when we compared the current study with an ethnobotanical study conducted in Cizre (Şırnak), we observed similarities between the local names of 13 taxa: Crataegus azarolus var. azarolus, Elaeagnus angustifolia, Ficus carica subsp. carica, Glycyrrhiza glabra, Gundelia tourneforti, Malva neglecta, Mentha longifolia, Pistacia terebinthus subp. palaestina, Rhus coriaria, Quercus brantii, Rhus coriaria, Rumex crispus and Urtica dioica. It is already known that the common language of these areas is Kurdish.

We further observed many similarities between the local names of plants from Yeşilli and those from bordering countries: Armenia (six taxa) [97], Hawraman (Iraq) (six taxa) [98] and Iraq (four taxa) [99].

Furthermore, almost 12 taxa (c.16\%) were also available at the public market and local shops. This shows that the plants are largely freshly harvested and consumed and the persistence and possibly economic viability of the domestic, small-scale wild vegetable trade.

\section{Differences among the three studied communities}

In this study, the number of interviewed Kurd informants was 39, Arabs was 18 and Assyrians was five. The consumption ratio of wild edible plants is as follows: Kurds (80.26\%), Assyrians (60.52\%) and Arabs (23.68\%). The most overlap of the obtained data and the Jaccard index (similarity) was between Kurds and Assyrians and the least one was between Arabs and Kurds, perhaps due to the different main ecological areas where wild edible plants were collected (Fig. 3). The Kurds, whose livelihood is horticulture and mainly husbandry, have maintained gathering wild edible plants around their villages and from high mountains for a long period of time. In Bülbül village (the Assyrian village), since people generally speak in Arabic, the Syriac names of the plants had mostly forgotten. Therefore, most of the plants' names were recorded in Arabic. Although fewer people were interviewed in the Assyrian village due to the law population, the use of many plants clearly indicates that they continue to use wild edible plants extensively and continue to hand down this traditional knowledge to the next generations. Assyrian beliefs have also an important role on consuming the plants. For instance, an informant who was educated in the Darülzefaran Monastery (an Assyrian Monastery) stated that parts with in the form of a cross are harmless and can be eaten or used. On the other hand, although more Arabs were interviewed than the Assyrians, less traditional knowledge was recorded. The reason for this is that because the Arabs live in the district center where there is higher capability to buy food. They often consume the plants that the Kurds gather from their villages and surrounding mountains and bring them to the public markets and local shops.

The most culturally important taxa were Ficus carica subsp. carica, Lepidium draba, Anchusa strigosa, Rhus coriaria, Glycyrrhiza glabra, Sinapis alba, Gundelia tournefortii, Notobasis syriaca, Onopordum carduchorum, Malva

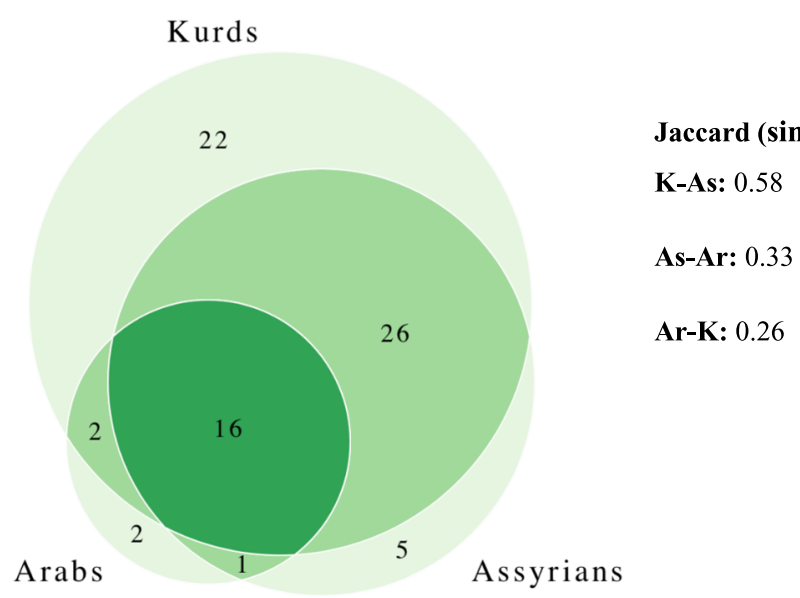

Fig. 3 Overlap in the wild edible plants traditionally gathered by three studied communities 
neglecta, Mentha longifolia, Juglans regia and Urtica dioica. However, these plants are popular among the three communities in different manners. In addition to these plants Olea europaea, Pistacia terebinthus subsp. palaestina and Capparis sicula which has low cultural index are also consumed by three communities. However, Anchusa strigosa, Gundelia tournefortii, Notobasis syriaca and Onopordum carduchorum were mainly mentioned by Assyrians and Kurds. While Rosa canina, R. foetida, Rubus sanctus, Crataegus azarolus var. azarolus, Crocus cancellatus subsp. damacenus and Thymbra spicata subsp. spicata with high cultural index prevailed only by Assyrians and Kurds, Arum rupicola and Erodium cicutarium were mentioned by Arabs and Kurds and Eryngium creticum were mentioned by Arabs and Assyrians.

On the other hand, Centaurea virgata, Echinophora tenuifolia subsp. sibthorpiana, Echinops spinosissimus subsp. bithynicus, Geocaryum cynapioides subsp. macrocarpum and Silene dichotoma were only mentioned by Assyrians and Salvia syriaca and Polygonum cognatum were only mentioned by Arabs. Twenty-two taxa were mentioned only by Kurds, among these species Amaranthus retroflexus, Elaeagnus angustifolia, Nasturtium officinale and Vicia sativa subsp. nigra were metioned particularly by many interviewees.

Producing herbal cheese is common among the Kurds living in high altitude villages, similar to the eastern and the southeast regions of Turkey [74]. The beverage called 'ava susê', prepared from roots of Glycyrrhiza glabra is also common among them. However, only the Assyrians use the fruits of Cerasus mahaleb var. mahaleb to produce liqueur and wine. Besides, it was recorded that the leaves of Arum rupicola were consumed by Arabs and Kurds.

\section{Informant consensus index $\left(\mathrm{F}_{\mathrm{ic}}\right)$}

The informant consensus index $\left(F_{i c}\right)$ varied between 0.99 for preserved vegetables and 0.95 for raw vegetables (Table 3). The $F_{i c}$ for beverages and spices was 0.98 . These results reveal that although different cultures and different environments are present in the study area, people continue to use wild food plants intensively.

Use categories and cultural importance of wild edible plants We classified the wild edible plants into eight categories. The category with the most plants was vegetables (46 taxa), followed by medicinal plants (17 taxa) and fruits (14 taxa) (Fig. 4). The CI index value of vegetables was $42.71 \%$ of the total CI, followed by medicinal WEPs $20.28 \%$, fruits $15.12 \%$ and beverages $7.10 \%$. The maximum number of use reports was recorded for vegetables (1011) (Table 3). The life forms of wild edible plant taxa include mainly herbs $(74.32 \%, 55$ taxa), followed by shrubs $(17.56 \%, 13$ taxa) and trees (8.1\%, 6 taxa) (Fig. 5). According to the CI index, the most important vegetable taxa were Lepidium draba (CI 0.96), Anchusa strigosa (CI: 0.88), Sinapis alba (CI: 0.83), Gundelia tournefortii (CI: 0.80), Notobasis syriaca (CI: 0.80), Onopordum carduchorum (CI: 0.80), Malva neglecta (CI: 0.77), Urtica dioica (CI: 0.72), Rosa foetida (CI: 0.65), Crocus cancellatus subsp. damascenus (CI: 0.61), Nasturtium officinale (CI: 0.50) and Amaranthus retroflexus (CI: 0.40). The most common fruit consumed by the locals were Ficus carica subsp. carica (CI: 0.96), Crataegus azarolus var. azarolus

Table 3 Wild edible plant taxa and their cultural importance of various use-categories and subcategories

\begin{tabular}{|c|c|c|c|c|}
\hline Use-category/subcategory & Number of species & The number of use reports (UR) & $\mathrm{Cl}$ & Fic \\
\hline Vegetables & 46 & 1011 & 16.30 & 0.96 \\
\hline Vegetable (raw) & 35 & 670 & 10.81 & 0.95 \\
\hline Vegetable (cooked) & 20 & 443 & 7.14 & 0.96 \\
\hline Vegetable (preserved) & 6 & 102 & 1.65 & 0.95 \\
\hline Fruits & 14 & 391 & 5.77 & 0.97 \\
\hline Fruit (raw) & 14 & 384 & 6.19 & 0.97 \\
\hline Fruit (processed) & 3 & 176 & 2.83 & 0.99 \\
\hline Seeds & 8 & 127 & 2.04 & 0.94 \\
\hline Seed (raw) & 7 & 109 & 1.75 & 0.94 \\
\hline Seed (cooked) & 2 & 53 & 0.85 & 0.98 \\
\hline Beverage & 5 & 168 & 2.71 & 0.98 \\
\hline Nectar & 3 & 74 & 1.19 & 0.97 \\
\hline Spices & 4 & 140 & 2.25 & 0.98 \\
\hline Latex & 2 & 10 & 0.16 & 0.89 \\
\hline Medicinal WEPs & 17 & 480 & 7.74 & 0.96 \\
\hline
\end{tabular}




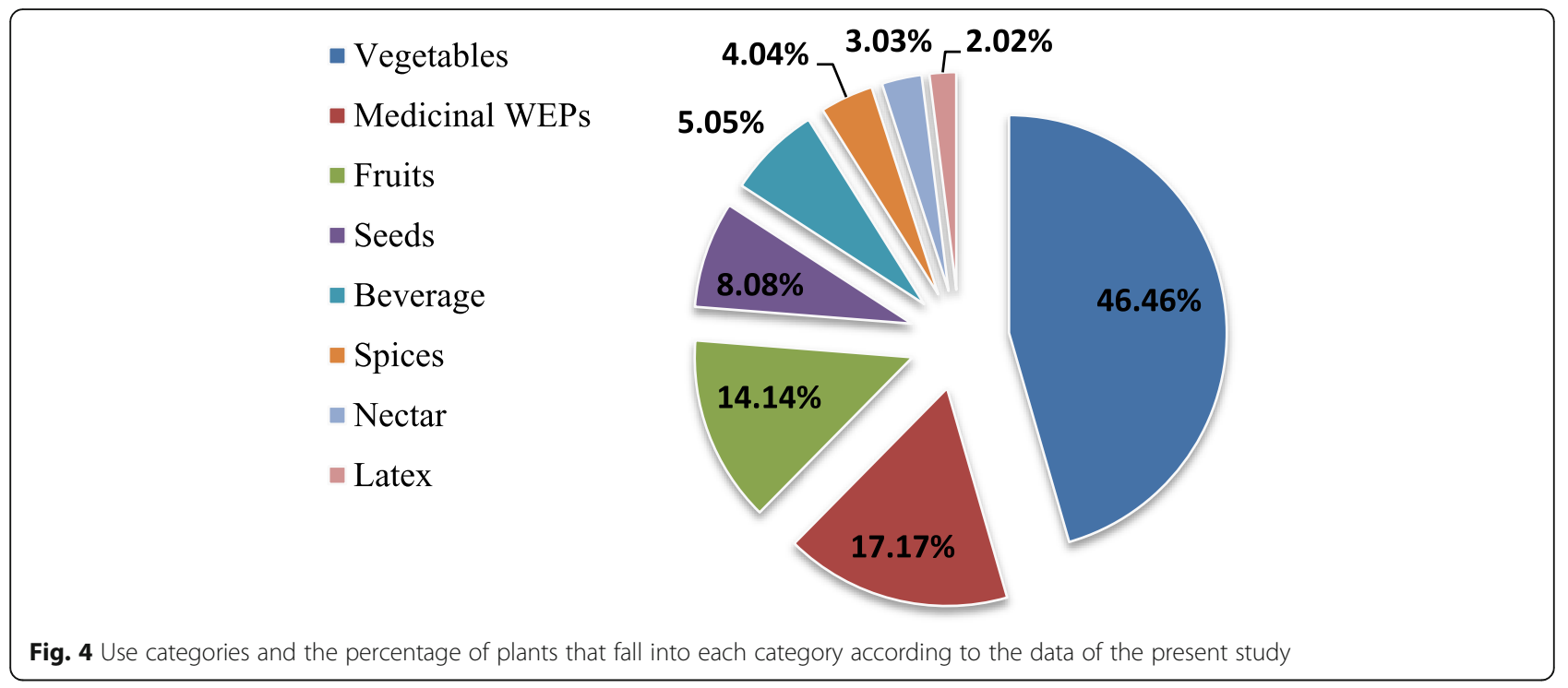

(CI: 0.61) Rubus sanctus (CI: 0.54), Rosa canina (CI: 0.53 ) and Pistacia terebinthus subsp. palaestina (CI: $0.48)$.

\section{Vegetables}

The intense, diverse use of wild vegetables was reported in East Turkey and near the border of Turkey [99]. The present study confirms previous results because most taxa with high CI index value were vegetables. While Sinapis $a l b a$ is a culturally important species in the study area, its usage is not very common in Turkey $[26,28,41,42,92$, 99]. Malva neglecta has substantial local value as a vegetable in Turkey and bordering countries $[26,28,30-34$, $36,38,40,44,47-49,51-55,59,62,65,76,78-82,84,88$, $89,94,98]$. Also it is a necessary plant in Yeşilli; the young aerial parts of Malva neglecta are boiled and then fried with onion. It is rich in vitamins A, B and C [110].
The tubers of Geocaryum cynapioides are peeled and eaten raw (Fig. 6). This plant is thought to have a brainlike appearance and is believed to be a tonic for the brain when consumed. The corms of Crocus cancellatus subsp. damascena and Gagea villosa are collected in early spring, then corm tunics are peeled and the fleshy part is eaten raw. The same use of Crocus cancellatus subsp. damascenus was previously reported in the southeast and eastern region of Turkey [26-28, 79, 94]. Gagea villosa is widely distributed in Turkey; however, the use of it has never been repoted to be used as. While the gall of Salvia syriaca is eaten raw in Yeșilli, as consumed in Kürecik [94], its fruits are consumed as spices in Midyat [31], flowers as herbal tea in Cizre [79].

The consumption of Centaurea hyalolepis leaves was recorded in the present study for first time. A previous study [26] reported that the flowers of the plant were eaten. Additionally, the consumption of Centaurea

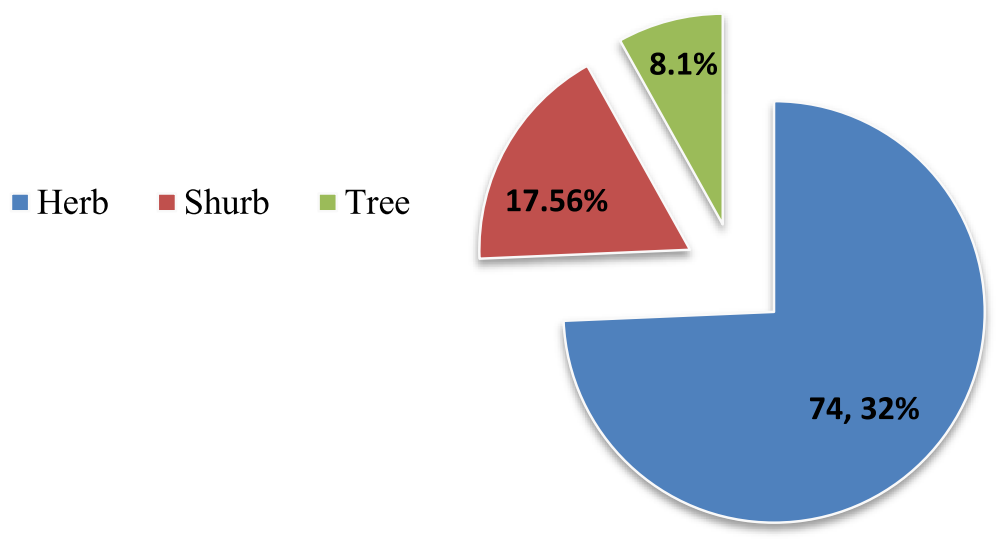

Fig. 5 Life forms of wild edible plants 


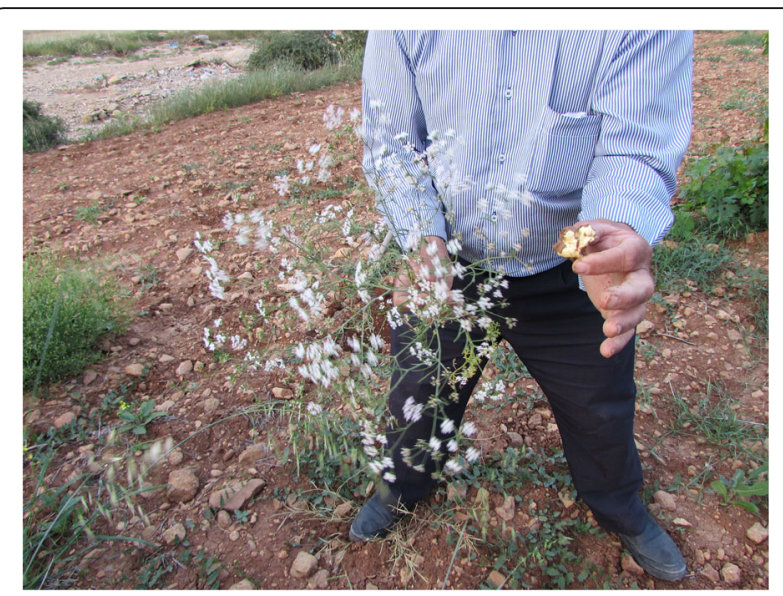

Fig. 6 Geocaryum cynapioides and its tuber

virgata as food was also recorded only in this study; this plant is named 'tal', which means 'hot'. The reason for this taste is the chemical compounds of the species, including sesquiterpenes, flavones and the flavonol isokaempferide [111]

Cooked plants are fried and then consumed or boiled and then a soup is prepared by adding yogurt and boiled bulgur. For instance, the aerial parts of Gundelia tournefortii, Lepidium draba, Sinapis alba, Urtica dioica and $U$. pilulifera are only fried. Additionally, the leaves of Allium ampeloprassum, stems of Gundelia tournefortii and the aerial parts of Amaranthus retroflexus, Anchusa strigose, Chenopodium album, Convolvulus arvensis, Malva neglecta and Polygonaum cognatum are boiled, added to yogurt and bulgur or boiled and sometimes fried with egg.

Although the consumption of Anchusa strigosa is common in the study area, the use of this plant as food is not common in Turkey [26, 31, 94] and the other regions that it distributed. The young leaves of Anchusa strigosa are first boiled and then fried with onion. The species is also used for medicinal purposes [112-116]. It contains alkaloids, tannins and vitamin E [117]. Young stems, roots and petioles of Gundelia tournefortii are consumed raw or cooked in the study area, as in the other parts of Turkey [26, 27, 31, 33, 40-42, 52, 55, 60, $65,70,72,78,80-82,84,86,89,94]$ and in bordering countries $[96,98,99,114,118]$. This species is a good source of vitamins A, C and E [119].

Two recent studies [44, 45] documented the taxa used for preparing 'Sarma' in Turkey and the Balkans. Sarma is a cooked leaf rolled around a filling made from rice and/or minced meat. Rumex obtusifolia subsp. subalpi$n u s$ and $R$. crispus are among the plants used for preparing sarma in the study area.

It is common to produce herbal cheese among the local people in the eastern and the southeast regions of Turkey, where ovine are bred and aromatic herbaceous plants are widely grown [74]. The leaves of Allium ampeloprassum, A. chloranthum and A. wendelboanum are eaten fresh or added to cheeses. To produce this herbal cheese, once the sheep milk is fermented and gelatinised, a coating of gel is placed in a cloth, a layer of milk is placed in the gel, a layer of finely divided chopped plant pieces is added, the bag is tightly wound and a weight is placed on the bag to drain water. Allium ampeloprassum is often used for the same purpose [60, 70, 74], but the use of Allium chloranthum and $A$. wendelboanum is not mentioned previously in the ethnobotanical studies in Turkey.

In the Mediterranean region and similarly in the study area, the immature flower buds of Capparis species are often used to prepare pickle [2, 3]. Also it is used as pickle and food in Cizre and Şanlıurfa and in some other regions of Turkey [27, 28, 41, 52, 56, 65].

Even though Hirschfeldia incana is broadly distributed in Turkey and was reported in an ethnobotanical study in Iran [120], its consumption was not previously reported. This species is consumed as Sinapis and Lepidium species.

Iris persica and I. reticulate are known as the heralds of the arrival of spring. Their flowers are eaten raw and have a mild taste (Fig. 7). The same consumption was reported previously for Iris persica [82, 94] and for Iris reticulate [82]. However, it was stated that the infusion prepared from flowers of Iris reticulate was consumed like tea in Midyat [31]. Also, the petals of Rosa foetida are eaten raw by children. The consumption of the flowers of this plant as food and for medicinal purposes has been recorded in Midyat [31], but there are no previous records of the use of flowers as food.

Previous studies reported that Arum spp. leaves were kept in an acidic environment (created using a powdered sour sumac spice mixed with water or cheese water) before the leaves were consumed to remove excess oxalates and sharp flavour found in the leaves [94, 96, 98, 99]. In the present study,

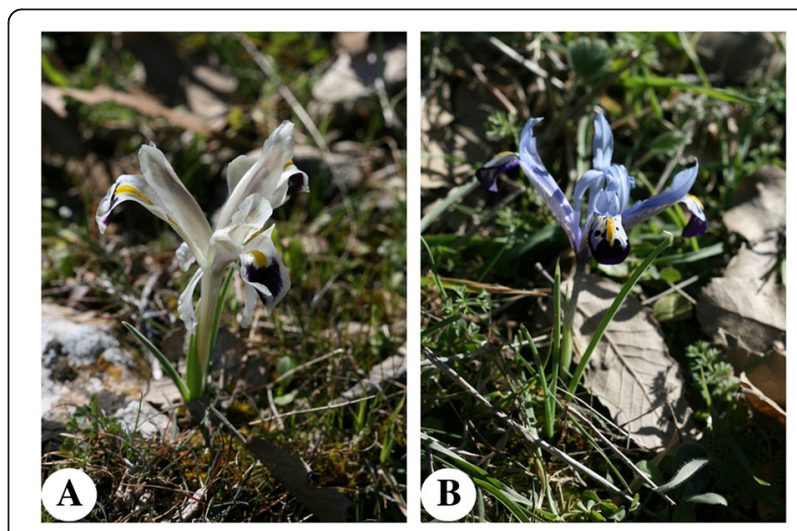

Fig. 7 Flowers of Iris species consumed by people in the present study. a Iris persica. b I. reticulata 
lemon salt and pomegranate juice were also mentioned in addition to sumac spice by the interviewees for detoxification of Arum rupicola leaves (Fig. 8). After this process, the boiled leaves are fried with onion.

Euphorbia species are often known as poisonous and they are not consumed directly. However, the aerial parts of Euphorbia helioscopia subsp. helioscopia are added to molasses white soil when it boils to improve the test of molasses. Also the consumption of this species was reported previously as food [41]. Moreover, it was reported that Euphorbia species were used to clean thick buble layer on grape molasses in Midyat [31], and were added to the jam and used to ferment cheese or dough in Cizre [52].

\section{Fruits}

The fruits of 13 taxa are consumed raw. However, the fruits of Celtis tournefortii, Ficus carica subsp. carica and F. carica subsp. rupestris are also dried and eaten in winter. The most common fruits consumed by informants were Celtis tournefortii, Cerasus prostrata, Crataegus azarolus var. azarolus, Elaeagnus angustifolia, Ficus carica subsp. carica, Rosa canina and Rubus sanctus. Ficus carica subsp. carica had the highest CI index in the study area; the consumption of this taxa is commonly reported $[20,26,37,38,40,43,50,52,55,56,58$, $59,62,63,72,75,82,86,92,96]$. The consumption of the fruits of Ficus carica subsp. rupestris is not common $[27,40,94]$. Rosa canina has high local value as a fruit in Turkey, as well as in bordering countries and European countries $[2-4,20,32-36,38,40,41,47-49,51-$ $53,55,60,62,63,65,69-72,74,75,78,81,82,86,93$, $94,96]$. The fruits of this species are well-known for their efficacy in strengthening the body's defence against infection and particularly the common cold [36]. Furthermore, fruits are rich in sugar, pectin, organic acids, essential oils, tannins and minerals $(\mathrm{P}, \mathrm{K}, \mathrm{Mg}, \mathrm{Ca}$,

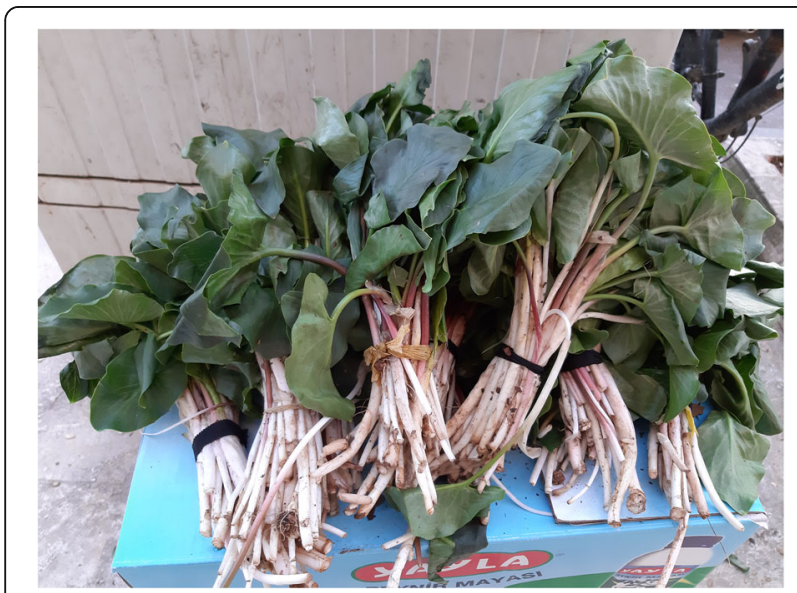

Fig. 8 Arum rupicola leaves in the local market in Yeşilli District
Fe, Mn) [121]. The fruit of Cerasus microcarpa subsp. microcarpa are eaten, and the same usage was observed in previous studies $[49,60,94]$. However, the consumption of Cerasus prostrata var. prostrata was not previously reported. The fruits of Celtis tournefortii are consumed freshly; additionally, the mature fruits have another interesting usage in the region. The fruits are fried, crushed and powdered and used to prepare a mixture with honey or molasses, which is usually eaten in winter. Celtis tournefortii fruits have important antiradical, antimicrobial and antiproliferative properties [122]. The similar use of Celtis glabrata Steven ex Planch. fruits has been previously reported [60].

\section{Seeds}

The seeds of five taxa from the Fabaceae family are eaten raw. The local name 'colban' is used for the fruits of Vicia and Lathyrus species in the southeast of Turkey [27]. However, these plants named 'baqil' in Yeșilli. Also, they are named according to the shapes of the fruits, seeds and leaves in the study area. Baqil is the common name of these species. Vicia palaestina is called baqilê çûkan; 'çûkan' means 'birds' and this name is used because the fruit of this plant is smaller than the fruit of other baqil species. Vicia pannonica is called baqilê gan; ' $g a n$ ' means ' $O x$ 's', and this name is used because the fruit of this plant is larger than the fruit of other baqil species. The usage of these taxa as only fodder was reported in Urfa [26]. Vicia pannonica var. pannonica is commonly used according to an ethnobotanical study conducted in Midyat district [31].

Vicia sativa is named baqilê maran; 'maran' means 'snake's' and this name is used because the fruit of the plant is longer and thinner than the fruit of other baqil species. The consumption of the fruits of Vicia sativa was previously reported [27, 49, 52, 63]. The seeds of this plant contain $31 \%$ crude protein, $327 \%$ starch, $54 \%$ sugar and 35-44\% fibre, cellulose and lignin [107].

Moreover, Lathyrus cassius is named baqilê bizinan; 'bizinan' means 'goat's', as the fruits and leaves of this plant are smaller than the fruit of Vicia pannonica. Pisum fulvum is named 'baqilê xatûnî'; 'xatûnî' means 'goddess's', the seeds of this plant are tastier than those of other baqil species. The consumption of Lathyrus cassius and Pisum fulvum were reported for the first time in this study.

The fruits of Quercus brantii were reported to be eaten raw and roasted in embers in the present study, but raw consumption is generally reported for medicinal purposes in Turkey [31, 40, 52, 61]. A previous study reported the same consumption in the southeast of Turkey [123].

\section{Beverage}

In the studied area, five taxa were reported to be used for preparing beverages such as herbal teas, coffees, liqueur or 
wine and cold drinks. The flowers of Elaeagnus angustifolia and the leaves of Mentha longifolia are prepared as a herbal tea. Immature fruits of Pistacia terebinthus subsp. palaestina are collected when they are purple and blue (in September). Then, these fruits are dried, crushed and cooked as coffee. This coffee is called melengiç, menengiç, çitlenbik, çedene, kizvan kahvesi and is prepared with water or milk. It is very common in Turkey's southeast region and is consumed daily as a drink [30, 38, 41-43, 49, $50,52,54,56,59,60,65,75,78,81,86,91]$. The Christian people use the fruits of Cerasus mahaleb var. mahaleb to prepare liqueur and wine. The roots of Glycyrrhiza glabra are used to prepare a traditional cold beverage called 'ava susê' during summer. To prepare ava susê, the collected roots are washed, cut to a length of $20 \mathrm{~cm}$, placed in a bowl and sprinkled with a small amount of water. Then, the mixture is kneaded like dough. When the water is absorbed, more water is added. This process is repeated several times. Additional water is added to the roots. The liquorice root water obtained is called yeast. A little more water is added to the yeast, and liquorice syrup is obtained (Fig. 9). To remove the bitter taste, the syrup is poured from one container to another and the foam is cleared. Ava susê has an important place in folk culture and there is even a Kurdish song about this drink in the region. The drink is distributed by itinerant vendors carrying large

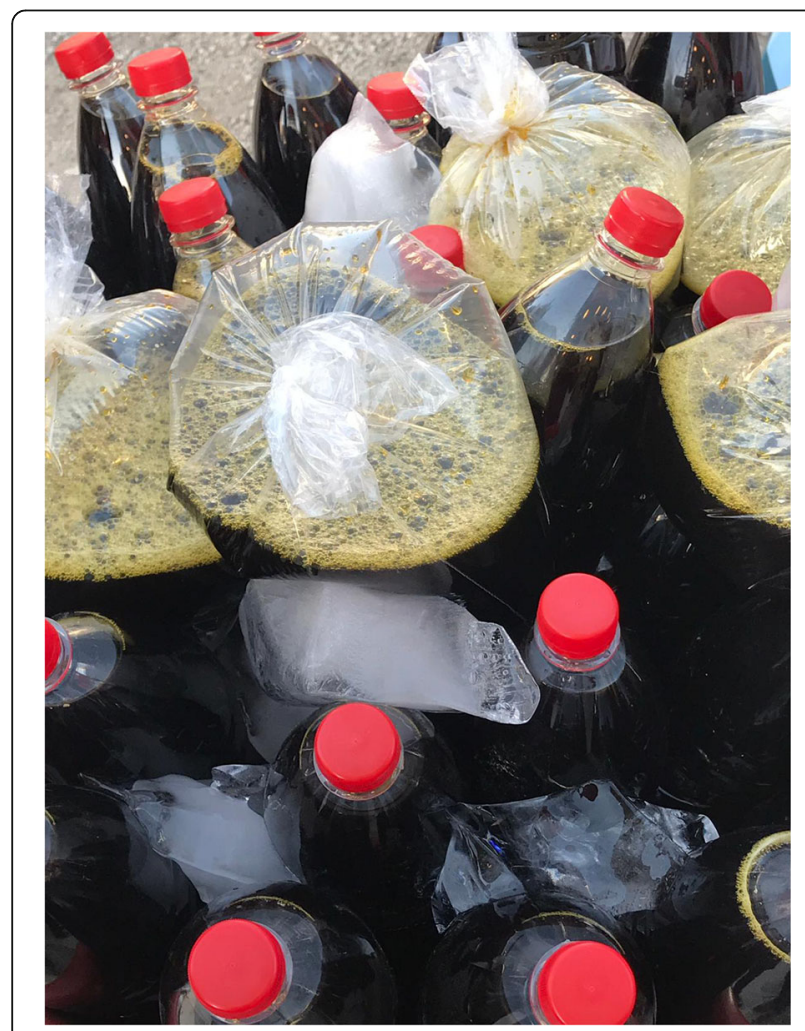

Fig. 9 Liquorice syrup obtained from Glycyrrhiza glabra roots in a shop in the center of Yeşilli decorated pitchers on their backs. The consumption of Glycyrrhiza glabra roots in this way was also reported in a previous study [12] and it is generally consumed in the eastern and the south-eastern parts of Anatolia [26, 27, 32, $41,48,52,78,80-82,93]$. The sweet taste of the beverage is due to the substances in it. Glycyrrhiza glabra contains flavonoids, isoflavones, saponins, coumarins, chalcones, sterols and alkaloids [124]; the main component glycyrrhizin $(2-25 \%$ content) is 50 times sweeter than sucrose [124]. The plant is used to treat colds [52, 77], respiratory tract diseases, flu, bronchitis, gastrointestinal diseases, smoking addiction [83] and diabetes [90], and as analgesic. Antioxidant, antimicrobial (especially against Helicobacter pylori), immunostimulant and anticancer effects have also been reported in this plant [124].

\section{Spices}

Because of their aromatic properties, species from the Lamiaceae family, including Mentha longifolia, Thymbra spicata and Thymus kotschyanus subsp. kotschyanus, are used as spices; the aerial parts of these species are collected in April and are dried in a shady place and used as spices. Mentha longifolia is a valuable spice in Turkey [32-34, 38, 40, 44, 47-49, 51-54, 58, 60, 62, 63, 65, 69, $70,72,74-76,78,80-82,84-89,94]$ and close regions of bordering countries [94, 96, 98, 99].

The mature fruits of Rhus coriaria are collected at the end of July or in the first days of August and dried in shady places. The dried fruits are then crushed. Rhus coriaria is usually used where it is distributed [20, 28 , $31,34,38,41,52,55,60,65,66,71,75,78,81,82,86$, 92, 96]. The common name of Rhus coriaria is sumac; this name derives from 'sumaga', which means red in Syriac [125]. It is used by indigenous peoples for medicinal purposes and other uses [126]. Different parts of sumac possess significant phytochemical components, such as tannins, flavonoids, anthocyanins, organic acids, flavones, proteins, fibre, volatile oils, nitrates and nitrites [127]. Furthermore, antimicrobial, antifungal and antiviral activities of Rhus coriaria were reported [127]. Thymbra spicata and Thymus kotschyanus subsp. kotschyanus are generally called cahter or cehter, and they are the leaves are collected before flowering time, dried in the shade place and added to the food as flavour and fragrance. In the literature study, it was observed that Thymus kotschyanus subsp. kotschyanus was consumed as spices especially in Eastern Anatolia [32, 60, $62,70,74,78,81,82]$. However, the use of Thymbra spicata was reported usally as spice in the Mediterranean and nearby regions $[28,30,31,38,43,55,74,78,86]$.

\section{Nectar and latex}

Boraginaceae is known to produce high-quality nectar, which is a sugar-rich solution [128]. Usually, the nectar 
of flowers of Anchusa and Echium species is sucked [26, 31, 94, 99]. However, we observed the sucking of the nectar of Onosma roussaei and O. alborosea for the first time (Fig. 10). The edible latex which flows through the stems of Echinops spinosissimus subsp. bithynicus and $E$. orientalis that flows through the stem of these taxa is known as 'helva' because of its sweet taste ('helva' means 'sweet' in Arabic) (Fig. 11). This consumption of receptacles is common [27, 40, 60, 70, 78, 94], but the consumption of dried latex has not been previously reported.

\section{Medicinal WEPs}

Approximately quarter of the gathered and consumed wild vegetables that were recorded in this study (most notably Anchusa strigosa, Cerasus mahaleb var. mahaleb, Mentha longifolia, Glycyrrhiza glabra, Malva neglecta, Rosa canina and Urtica dioica) are also used for medicinal purposes; these plants are called nutraceuticals folk functional foods [12]. In some interviews, it was recorded that every eaten plant is believed to be good for health. In the present study, 16 wild edible taxa were reported as food medicine, including wild vegetables, roots and fruits. The aerial parts of Urtica dioica (CI: 0.72) are cooked as vegetables and consumed for the treatment of cancer, rheumatism and diabetes. Urtica dioica is a multi-purpose medicinal species [129]; it is consumed in addition to the fruits of Rosa canina (CI: 0.53) for the treatment of colds,

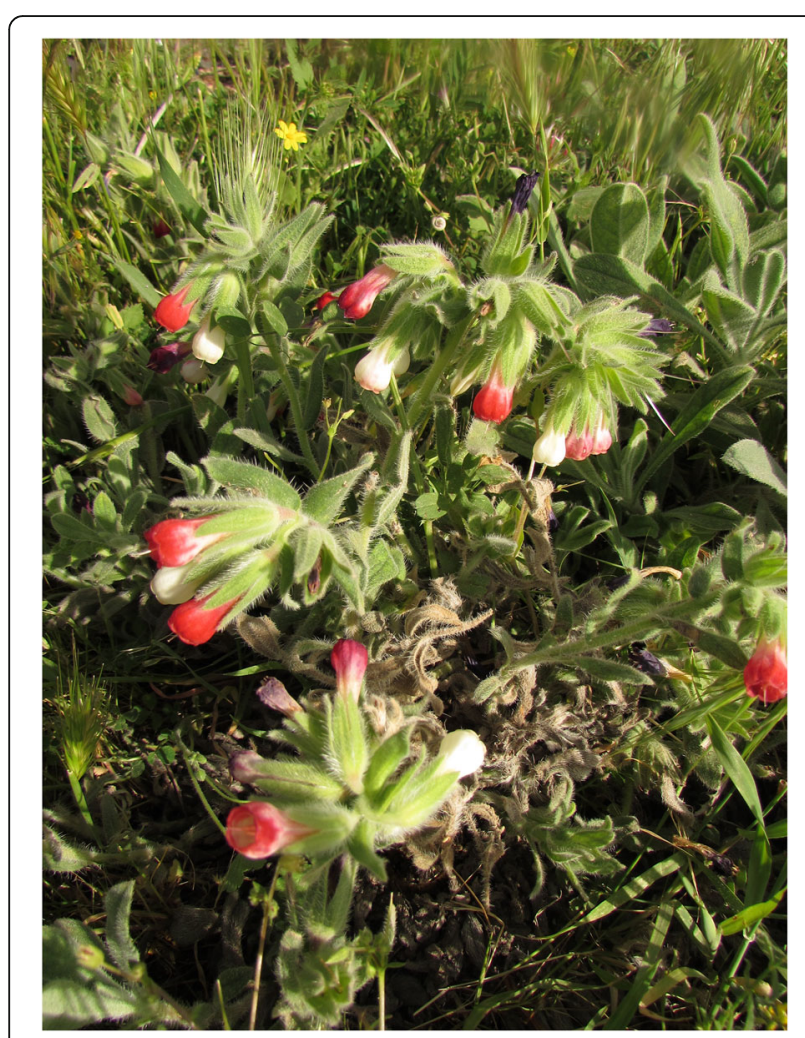

Fig. 10 Onosma alborosea

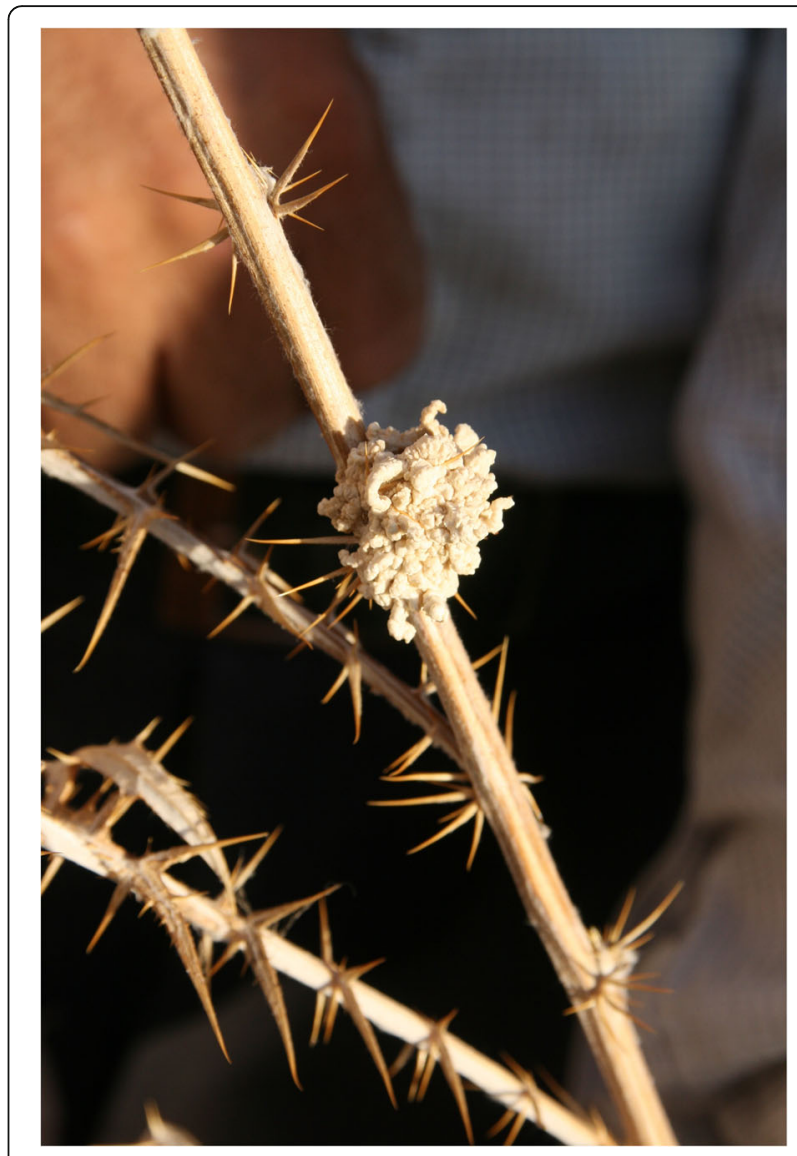

Fig. 11 The latex of Echinops orientalis

flu, cough, urinary tract burning and bronchitis. Urtica dioica is a rich source of flavonoids, carotenoids and fatty acids and has a high content of vitamins (especially vitamin C), minerals (Ca, Mg, Fe, $\mathrm{Al}, \mathrm{Mn}, \mathrm{Zn}, \mathrm{Sr}, \mathrm{Ba}$, etc.) and antiinflammatory agents [130]. Additionally, some parts of this medicinal plant and other plants (Cerasus mahaleb var. mahaleb, Thymbra spicata and Quercus brantii) are collected by local people, and sold in the local bazaars.

\section{Conclusion}

A comparison of the traditional uses of wild edible plants reported, in the literature, revealed that the current study is the first to record the use of Allium chloranthum, A. wendelboanum, Centaurea virgata, Cerasus prostrata var. prostrata, Gagea villosa, Geocaryum cynapioides, Lathyrus cassius, Onosma alborosea, Papaver glaucum, Pisum fulvum, Rosularia radiciflora and Sedum pallidum in Turkey and bordering countries. The culturally important plants in the study area (Ficus carica subsp. carica, Gundelia tournefortii, Glycyrrhiza glabra, Juglans regia, Lepidium draba, Malva neglecta, Mentha longifolia, Rhus coriaria, Rosa canina, Rumex crispus, and Urtica dioica) are also commonly used in other parts of Turkey and bordering countries near the 
study area, which shows that the residents of these regions have some similarities in their traditions.

This study in Yeşilli district, which is culturally diverse, and this area has not been subject to any ethnobotanical work previously. In multicultural and multilingual area, there are differences in plant usage as well as in plant names. Therefore, this study has made an important contribution to the preservation of cultural heritage related to traditional wild edible plants in this region. The villagers continue to agriculture and animal husbandry intensively. However, a large part of the population consists of elderly people, middle-aged people and children. The older generations of villagers have extensive knowledge of useful plants, and this knowledge is transmitted orally from one generation to another. Most of the young population works in large cities. Unfortunately, the few young people living in the villages are not interested in learning traditional knowledge from their ancestors. Hence, there is an urgent need to document their folklore, before traditional knowledge becomes unattainable and eventually extinct. Our findings will contribute to increasing the existing knowledge of wild edible plants in Mardin and its surroundings and will be a source for comparative intercultural ethnobotany.

\section{Acknowledgements}

Special thanks to Hamza Yılmaz, Eser Bozan and Abdullah Demir for help with fieldwork. We also thank all participants in the survey in Yeşilli District.

\section{Authors' contributions}

YY, BY and MÇ carried out fieldworks, collected the data and identified the plants, YY analysed the data and wrote the manuscript. All authors read and approved the final manuscript.

\section{Funding}

This study founded by Istanbul University Scientific Research Projects Department with 41403 coded project.

\section{Availability of data and materials}

All data generated or analysed during the conduct and writing up of the manuscript is incorporated in the research article. Voucher specimens were deposited at the Herbarium of Faculty of Pharmacy, University of Istanbul (ISTE).

\section{Ethics approval and consent to participate}

Prior informed consent was always verbally obtained before each interview and the Code of Ethics of the International Society of Ethnobiology (ISE 2008) was strictly followed [131].

\section{Consent for publication}

Not applicable.

\section{Competing interests}

The authors declare that they have no competing interests.

Received: 31 March 2019 Accepted: 4 September 2019

Published online: 05 November 2019

\section{References}

1. Addis G, Urga K, Dikasso D. Ethnobotanical study of edible wild plants in some selected districts of Ethiopia. Hum Ecol. 2005;33(1):83-118. https://doi. org/10.1007/s10745-005-1656-0.
2. Tardío J, Pardo de Santayana M, Morales R. Ethnobotanical review of wild edible plants in Spain. Bot J Linn Soc. 2006;152:27-71 https://doi.org/10. 1111/j.1095-8339.2006.00549.x.

3. Łuczaj Ł. Ethnobotanical review of wild edible plants of Slovakia. Acta Soc Bot Pol. 2012;81(4):259-370 https://doi.org/10.5586/asbp.2012.030.

4. Łuczaj Ł, Pieroni A, Tardio J, Pardo-de-Santayana M, Sõukand R, Svanberg I, Kalle R. Wild food use in 21st Century Europe: the dissapearance of old traditions and the search for new cuisines involving wild edibles. Acta Soc Bot Pol. 2012;81(4):245-55 https://doi.org/10.5586/asbp.2012.031.

5. Bonet MÀ, Vallès J. Use of non-crop food vascular plants in Montseny biosphere reserve (Catalonia, Iberian Peninsula). Int J Food Sci Nutr. 2002; 53(3):225-48 https://doi.org/10.1080/09637480220132841.

6. Heinrich M, Müller WE, Galli C, editors. Local Mediterranean food plants and nutraceuticals. Basel: Karger; 2006. p. 18-74. https://doi.org/10.1159/000095206

7. Łuczaj $Ł$. Changes in the utilization of wild green vegetables in Poland since the 19th century: a comparison of four ethnobotanical surveys. J Ethnopharmacol. 2010;128(2):395-404 https://doi.org/10.1016/j.jep.2010.01.038.

8. Parto-de-Santaya M, Tardío J, Blanco E, Carvalho AM, Lastra JJ, Miguel ES, Morales R. Traditional knowledge of wild edible plants used in the North west of the Iberian Peninsula (Spain and Portugal): a comparative study. J Ethnobiol Ethnomed. 2007;3:27 https://doi.org/10.1186/1746-4269-3-27.

9. Pieroni A. Gathered wild food plants in the upper valley of the Serchio river (Garfagnana). Central Italy. Econ Bot. 1999;53:327-41 https://doi.org/10.5586/ asbp.3519.

10. Pieroni A. Evaluation of the cultural significance of wild food botanicals traditionally consumed in Northwestern Tuscany. Italy. J Ethnobiol. 2001;21:89-104.

11. Pieroni A, Nebel S, Quave C, Münz H, Heinrich M. Ethnopharmacology of liakra: traditional weedy vegetables of the Arbëreshë of the Vulture area in southern Italy. J Ethnopharmacol. 2002;81(2):165-85 https://doi.org/10.1016/ S0378-8741(02)00052-1.

12. Pieroni A, Nebel S, Santoro RF, Heinrich M. Food for two seasons: culinary uses of non-cultivated local vegetables and mushrooms in a south Italian village. Int J Food Sci Nutr. 2005;56(4):245-72 https://doi.org/10.1080/ 09637480500146564

13. Davis PH, editor. Flora of Turkey and the East Aegean Islands, vol. 1-9. Edinburgh: University Press; 1965-1985.

14. Davis PH, Mill RR, Tan K. Flora of Turkey and the East Aegean Islands. Vol. 10 (Supplement I). Edinburgh: University Press; 1988.

15. Güner A, Özhatay N, Ekim T, Başer KHC. Flora of Turkey and the East Aegean Islands. Vol. 11 (Supplement II). Edinburg: University Press; 2000.

16. Güner A, Aslan S, Ekim T, Vural M, Babaç MT. The checklist of Flora of Turkey (Vascular Plants). İstanbul: Flora Araştırmaları Derneği ve Nezahat Gökyiğit Botanik Bahçesi Yayını (in Turkish); 2012

17. Güner A. Illustrated Flora of Turkey Vol. 1. İstanbul: Türkiye İ̧̧ Bankası Kültür Yayınları (in Turkish); 2014.

18. Güner A, Kandemir A, Menemen Y, Yıldırım H, Aslan S, Ekşi G, Güner I, Çimen AÖ. Illustrated Flora of Turkey Vol. 2. İstanbul: ANG Vakfı Nezahat Gökyiğit Botanik Bahçesi Yayınları (in Turkish); 2018.

19. Özhatay N, Kültür \$̧, Gürdal B. Chek-list of additional taxa to the supplement flora of Turkey VIII. Istanbul J Pharm. 2017;47(1):30-44 https://doi.org/10. 5152/IstanbulJPharm.2017.006.

20. Bulut G. Medicinal and wild food plants of Marmara Island (Balıkesir-Turkey). Acta Soc Bot Pol. 2016:85(2):1-16 https://doi.org/10.5586/asbp.3501.

21. Ertuğ F. Etnobotanik. In: Güner A, editor. Resimli Türkiye Florası Cilt 1. Istanbul: Türkiye Iss Bankası Kültür Yayınları; 2014.

22. Kendir G, Güvenç A. Etnobotanik ve Türkiye'de yapıımış etnobotanik çalışmalara genel bir bakış. Hacettepe Univ Eczacı Fak Derg. 2010;30(1):49-80.

23. Tuzlacı E. Wild food plants and herbs dishes of Turkey. İstanbul: Alfa Yayınları (in Turkish); 2011.

24. Urhan Y, Ege MA, Öztürk B, Elgin CG. Turkish food plants database. J Fac Pharm Ankara. 2016;40(2):43-57 https://doi.org/10.1501/Eczfak_0000000583.

25. Akalın E, Alpınar K. Tekirdağ'ın tıbbi ve yenen bitkileri hakkında bir araştırma. Journal of pharmacy Ege University. 1994;2:1-11.

26. Akan H, Korkut MM, Balos MM. An ethnobotanical study around Arat Mountain and its surroundings (Birecik Sanlıurfa). Sci Eng J Firat Univ. 2008; 20(1):67-81.

27. Akan H, Balos MM, Tel AZ. Etnobotanical properties of some Leguminous plants in Birecik (Sanliurfa) region. ADYÜTAYM. 2013;1(1):31-9.

28. Akan H, Ayaz H. Flora of Gölpınar (Şanlıurfa, Turkey) excursion spot and ethnobotany properties of the surrounding villages. J. Bağbahçe Bilim. 2015; 2(3):19-56. 
29. Akan H, Bakır SY. The investigation of the Kâhta (Adıyaman) center and Narince Village from the point of ethnobotany. BEÜ Fen Bilimleri Dergisi. 2015;4(2):219-48

30. Akaydın G, Şimşek I, Arıtuluk ZC, Yeşilada E. An ethnobotanical survey in selected towns of Mediterranean subregion (Turkey). Turk J Biol. 2013;37: 230-47. https://doi.org/10.3906/biy-1010-139.

31. Akgül A, Akgül $A$, Şenol SG, Yıldırım H, Seçmen Ö, Doğan Y. An ethnobotanical study in Midyat (Turkey), a city on the silk road where cultures meet. J Ethnobiol Ethnomed. 2018;14:12 https:/doi.org/10.1186/s13002-017-0201-8.

32. Altundağ ÇE. Traditional knowledge of wild edible plants of Iğdır Province (East Anatolia, Turkey). Acta Soc Bot Pol. 2017;86(4):3568 https://doi.org/10.5586/asbp.3568.

33. Arı S, Temel M, Kargıoğlu M, Konuk M. Ethnobotanical survey of plants used in Afyonkarahisar-Turkey. J Ethnobiol Ethnomed. 2015;11:84 https://doi.org/ 10.1186/s13002-015-0067-6.

34. Bağcı Y. Ethnobotanical properties of Aladaglar (Yahyali, Kayseri) and its surroundings. Herb J. Syst. Bot. 2000;7(1):89-94.

35. Baykal $H$, Atamov V. Ethnobotanical documentation of plants of Başhemşin Valley, Kaçkar Mountains National Park, Rize, Turkey. Bangladesh J. Bot. 2017;46(2):767-73.

36. Baytop T. Herbal medicine in Turkey, past and present. İstanbul: Nobel Tıp Kitabevleri Yayınları (in Turkish); 1999.

37. Bulut G, Tuzlacı E. Useful plants and flora of Bozcada. İstanbul: Bozcaada Kaymakamlığı (in Turkish); 2009.

38. Bulut G, Tuzlaci E. Useful plants and flowers of Turgutlu. Manisa: Turgutlu Ticaret ve Sanayi Odası (in Turkish); 2015.

39. Doğan A, Bulut $G$, Şenkardeş I, Tuzlacı E. A review of edible plants on the Turkish Apiaceae species. J Fac Pharm. 2014;44(2):251-62.

40. Doğan A, Tuzlacı E. Wild edible plants of Pertek (Tunceli - Turkey). Marmara Pharm J. 2015;19:126-35 https://doi.org/10.12991/mpj.20151910459.

41. Doğan Y, Başlar S, Mert HH, Ay G. The use of wild edible plants in Western and Central Anatolia (Turkey). Econ Bot. 2004;58(4):684-90 https://doi.org/ 10.1663/0013-0001(2004)058[0684:TUOWEP]2.0.CO;2.

42. Doğan Y. Traditionally used wild edible greens in the Aegean Region of Turkey. Acta Soc Bot Pol. 2012;81(4):329-42 https://doi.org/10.5586/ asbp.2012.037.

43. Doğan Y, Uğulu I, Durkan N. Wild edible plants sold in the local markets of Izmir, Turkey. Pak J Bot. 2013;45(SI):177-84.

44. Doğan Y, Nedelcheva A, Luczaj L, Drăguluscu C, Stefkov G, Maglajlić A, et al. Of the importance of a leaf: the ethnobotany of sarma in Turkey and the Balkans. J Ethnobiol Ethnomed. 2015;11:26 https://doi.org/10. 1186/s13002-015-0002-X.

45. Doğan $Y$, Nedelcheva A, Pieroni A. The diversity of plants used for the traditional dish sarma in Turkey: Nature, garden and traditional cuisine in the modern era. Emir J Food Agric. 2017;29:429-40 https://doi.org/10.9755/ ejfa.2016-09-1238.

46. Ecevit Genç G, Özhatay N. An ethnobotanical study in Çatalca (European part of Istanbul). Turk Journ Pharm Sci. 2006;3:73-89.

47. Elçi B, Erik S. Ethnobotanical properties of Güdül (Ankara) and surrounding Hacettepe Univ Eczacı Fak Der. 2006;26(2):57-64.

48. Eren SB, Ozturk B, Sahin B, Senol S. The medical plants used around the world heritage site Mount Nemrut. Adiyaman (Turkey): XI International Ethnobotany Symposium Conference Proceedings; 2013. p. 79.

49. Ertuğ F. An ethnobotanical study in Central Anatolia (Turkey). Econ Bot. 2000;54(2):155-82 https://doi.org/10.1007/BF02907820.

50. Ertuğ F. Wild edible plants of the Bodrum area (Muğla, Turkey). Turk J Bot. 2004;28:161-74.

51. Everest A, Öztürk E. Focusing on the ethnobotanical uses of plants in Mersin and Adana provinces (Turkey). J Ethnobiol Ethnomed. 2005;1:6 https://doi. org/10.1186/1746-4269-1-6.

52. Gençay F. Cizre (Şırnak)'nin etnobotanik özellikleri [Ethnoboanical spects of Cizre (Şırnak)], MSc thesis. Van: Yüzüncü Yıl University; Department of Biology; 2007.

53. Gümüş I. Ağrı yöresinde yetişen bazı faydalı bitkilerin yerel adları ve kullanılışları. Turk J Bot. 1994;18:107-12.

54. Güneş F, Özhatay N. An ethnobotanical study from Kars (Eastern) Turkey. Biol. Diversity Conservation. 2011;4(1):30-41.

55. Güneş S, Savran A, Paksoy Yavuz M, Çakılcıoğlu U. Survey of wild food for human consumption in Karaisalı (Adana-Turkey). Indian J Tradit Know. 2018; 17(2):290-8 http://nopr.niscair.res.in/handle/123456789/43649.

56. Gürdal B, Kültür \$̧. The edible and miscellaneous useful plants in Marmaris (Southwest of Turkey). J Fac Pharm. 2014;44(1):69-78.
57. Kargıoğlu M, Cenkci S, Serteser A, Evliyaoğlu N, Konuk M, Kök MŞ, et al. An ethnobotanical survey of inner-west Anatolia, Turkey. Hum Ecol. 2008;36: 763-77 https://doi.org/10.1007/s10745-008-9198-x.

58. Karlıoğlu M, Cenkçi S, Serteser A, Konuk M, Vural G. Traditional uses of wild plants in the Middle Aegean region of Turkey. Hum Ecol. 2010;38:429-50 https://doi.org/10.1007/s10745-010-9318-2.

59. Kayabaşı NP, Tümen G, Polat R. Wild edible plants and their traditional use in the human nutrition in Manyas (Turkey). Indian J Tradit Know. 2018;17(2): 299-306 http://nopr.niscair.res.in/handle/123456789/43648.

60. Kaval İ, Behçet L, Çakilcioğlu U. Survey of wild food plants for human consumption in Geçitli (Hakkâri, Turkey). Indian J Tradit Know. 2015;14(2): 183-90 http://hdl.handle.net/123456789/32069.

61. Kılıç Ö, Bağcı E. An ethnobotanical survey of some medicinal plants in Keban (Elazığ - Turkey). J Med Plant Res. 2013;7(23):1675-84 https://doi.org/ 10.5897/JMPR2013.4451.

62. Kılıç Ö. An ethnobotanical survey from Bingöl (Turkey). RAJAR. 2016;2(10): 685-91.

63. Kızılarslan Ç, Özhatay N. An ethnobotanical study of the useful and edible plants in Izmit. Marmara Pharm J. 2012;16:194-200 https:/doi.org/10.12991/201216398.

64. Koca AD, Yıldırımlı Ş. Ethnobotanical properties of Akçakoca district in Düzce (Turkey). Hacettepe J. Biol. \& Chem. 2010;38(1):63-9.

65. Koçak S, Özhatay N. Wild edible plants in Karaman (Southern Turkey). Istabul J Pharm. 2013:43(1):21-32.

66. Koçyiğit M, Özhatay N. The wild edible and miscellaneous useful plants in Yalova Province (Northwest Turkey). Istabul J Pharm. 2008-2009;40:19-29.

67. Korkmaz M, Alpaslan Z, Turgut N, Ilhan V. Ethnobotanical aspects of some geophytes from Ergan Mountain, Turkey. Bangladesh J Bot. 2014;43(3):31521 https://doi.org/10.3329/bjb.v43i3.21604

68. Korkmaz M, Karakuş S, Selvi S, Çakılcıoğlu U. Traditional knowledge on wild plants in Üzümlü (Erzincan-Turkey). Indian J Tradit Know. 2016;15(4):538-45 http://nopr.niscair.res.in/handle/123456789/35229.

69. Kültür \$.. An ethnobotanical study of Kırklareli (Turkey). Phytol. Balcan. 2008; 14(2):279-89.

70. Mükemre $M$, Behçet $L$, Çakılcıoğlu U. Survey of wild food plants for human consumption in village of Çatak (Van-Turkey). Indian J Tradit Know. 2016; 15(2):183-91 http://hdl.handle.net/123456789/33966.

71. Nacakçı FM, Dutkuner I. A study of ethnobotany in Kumluca (Antalya). Turk J For. 2018;19(2):113-9 https://doi.org/10.18182/tjf.421970.

72. Nadiroğlu M, Behçet $L$. Traditional food uses of wild plants among the Karlıova (Bingöl-Turkey). Int J Nate Life Sci. 2018;2(2):57-71.

73. Özbucak TB, Kutbay HG, Akçin OE. The contribution of wild edible plants to human nutrition in the Black Sea region of Turkey. Ethnobot leaflets. 2006; 10:98-103.

74. Özçelik H. On the herbal cheese from East Anatolia (Turkey). Econ Bot. 1994; 48(2):214-7

75. Özdemir E, Kültür Ş. Wild edible plants of Savaştepe district (Balıkesir, Turkey). Marmara Pharm J. 2017;21(3):578-89 https://doi.org/10.12991/ marupj.319328.

76. Özgen U, Kaya Y, Coşkun M. Ethnobotanical studies in the villages of the district of Ilıca (province Erzurum), Turkey. Econ Bot. 2004;58(4):691-6 https://doi.org/10.1663/0013-0001(2004)058[0691:ESITVO]2.0.CO;2.

77. Özgökçe F, Özçelik H. Ethnobotanical aspects of some taxa in East Anatolia, Turkey. Econ Bot. 2004;58(4):697-704 https://doi.org/10.1663/00130001(2004)058[0697:EAOSTI]2.0.CO;2.

78. Öztürk M, Özçelik M. Useful plants of East Anatolia. Siirt: Siirt Ilim, Spor, Kültür ve Araştırma Vakfı (in Turkish); 1991.

79. Öztürk N, Dinç M. Ethnobotanical properties of Nizip (Aksaray) Region. Herb J. Syst. Bot. 2005;12(1):93-102.

80. Özüdoğru B, Akaydın G, Erik S, Yeşilada E. Inferences from an ethnobotanical field expedition in the selected locations of Sivas and Yozgat provinces (Turkey). J Ethnopharmacol. 2011;137:85-98 https://doi.org/10.1016/j.jep. 2011.04.050.

81. Polat R, Çakılcıoğlu U, Ulusan MD, Paksoy MY. Survey of wild food plants for human consumption in Elazığ (Turkey). Indian J Tradit Know. 2015;1(1):6975 http://hdl.handle.net/123456789/32029.

82. Polat R, Güner B, Babacan EY, Çakılcıoğlu U. Survey of wild food plants for human consumption in Bingöl (Turkey). Indian J Tradit Know. 2017;16(3): 378-84 http://nopr.niscair.res.in/handle/123456789/42023.

83. Sargın SA, Akçiçek E, Selvi S. An ethnobotanical study of medicinal plants used by the local people of Alaşehir (Manisa) in Turkey. J Ethnopharmacol. 2013;150:860-74 https://doi.org/10.1016/j.jep.2013.09.040. 
84. Sarper F, Akaydın G, Şimşek I, Yeşilada E. An ethnobotanical field survey in the Haymana district of Ankara province in Turkey. Turk J Biol. 2009:33:7988. https://doi.org/10.3906/biy-0808-28.

85. Sayar A, Güvensen A, Özdemir F, Öztürk M. Ethnobotanical properties of species in Mugla (Turkey) province. Herb J. Syst. Bot. 1995;2(1):151-60.

86. Şenkardeş A, Tuzlacı E. Some ethnobotanical notes from Gündoğmuş district (Antalya/Turkey). Marmara Üniversitesi Sağlık Bilimleri Enstitüsü Dergisi. 2014;4(2):63-75 https://doi.org/10.5455/musbed.20140303070652.

87. Şenkendareş I, Tuzlacı E. Wild edible plants in southern part of Nevşehir in Turkey. Marmara Pharm J. 2016;20:34-43 https://doi.org/10.12991/mpj.20163435871.

88. Şimşek I, Aytekin F, Yeşilada E, Yıldııımlı Ş. A research on the purpose and use of wild plants in Gölbaşı. Ankara. Herb J. Syst. Bot. 2001;8(2):105-20.

89. Şimşek I, Aytekin F, Yesilada E, Yildirimli Ş. An ethnobotanical survey of the Beypazari, Ayasand Güdül districttowns of Ankara Province (Turkey). Econ Bot. 2004 58(4):705-20 https:/doi.org/10.1663/0013-0001(2004)058[0705:AESOTB]2.0.CO;2

90. Tuzlacı E. Flora of Datça Peninsula (Muğla) and useful plants in this region: 14th Herbal Pharmaceutical Raw Materials Meeting Proceedings; 2004. p. 101.

91. Tonbul S, Altan Y. Some plants used by people for various purposes in Elazığ region. Science and Eng. J of Fırat Univ. 1989;3(2):267-78.

92. Uysal I, Onar S, Karabacak E, Çelik S. Ethnobotanical aspects of Kapıdac̆ Peninsula (Turkey). Biol Diversity Conservation. 2010;3(3):15-22.

93. Vural M, Karavelioğulları FA, Polat H. Ethnobotanical properties of Çiçekdağı (Kırşehir) and its surroundings. Herb J Syst Bot. 1997;4(1):117-24.

94. Yeşil Y, Akalın E. The use of edible plants in Kürecik (Akçadağ/Malatya). J Fac Pharm. 2010:41:90-103.

95. Abdalla M. Wild growing plants in the cuisine of modern Assyrians in the Eastern Syrian Turkish borderland. J Assyrian Academic Studies. 2004;18:50-8.

96. Ahmad SA, Askari AA. Ethnobotany of the Hawraman region of Kurdistan Iraq. Harvard Papers in Botany. 2015;20:85-9 https://doi.org/10.1186/s.13002-016-0081-3.

97. Hovsepyan R, Stepanyan-Gandilyan N, Melkumyan H, Harutyunyan L. Food as a marker for economy and part of identity: traditional vegetal food of Yezidis and Kurds in Armenia. Journal of Ethic Foods. 2016;3:32-41.

98. Pieroni $\mathrm{A}$, Ahmed $\mathrm{HM}$, Zahir $\mathrm{H}$. The spring has arrived: traditional wild vegetables gathered by Yarsanis (Ahl-e Haqq) and Sunni Muslims in Western Hawraman, SE Kurdistan (Iraq). Acta Soc Bot Pol. 2017:86:3519 https://doi.org/10.5586/asbp.3519.

99. Pieroni A, Sõukand R, Amin HIM, Zahir H, Kukk T. Celebrating multi-religious co-existence in Central Kurdistan: the bio-culturally diverse traditional gathering of wild vegetables among Yazidis, Assyrians, and Muslim Kurds. Hum. Ecol. 2018;46:217-27 https://doi.org/10.1007/s10745-018-9978-x.

100. Toprak L. Mardin ve Yemek Kültürü. Turkey, Mardin: Artuklu Yayınları; 2015.

101. Öztürkatalay L. Mardin ve Mardinliler. Seçil OFSET: Turkey, Istanbul; 1995.

102. Mardin Governor: http://mardin.gov.tr/ilcelerimiz/yesilli. Accesed 2 Jan 2019.

103. Merkel A. Climate-data. https://tr.climate-data.org/location/15560 (2016). Accesed 17 Dec 2018.

104. The Plant List. 2013. http://www.theplantlist.org. Accesed 21 June 2019.

105. Tardío J, Parto-de-Santaya M. Cultural importance indices: a comparative analysis based on the useful wild plants of southern Cantabria (northern Spain). Econ Bot. 2008;62(1):24-39 https://doi.org/10.1007/s12231-007-9004-5.

106. Jaccard P. Nouvelles recherches sur la distribution florale. Bull Soc Vaudoise Sci Nat. 1908:44:223-70.

107. Rebolé A, Alzueta C, Ortiz LT, Barro C, Rodríguez ML, Caballero R. Yields and chemical composition of different parts of the common vetch at flowering and at two seed filling stages. Span J Agric Res. 2004;2(4):550-7.

108. Bhatia H, Sharma YP, Manhas RK, Kumar K. Traditionally used wild edible plants of district UD hampur, J\&K, India. J Ethnobiol Ethnomed. 2018;14:73 https://doi.org/10.1186/s13002-018-0272-1.

109. Ojelel S, Mucunguzi P, Katuura E, Kakudidi EK, Namaganda M, Kalema J. Wild edible plants used by communities in and around seleceted forest reserves of Teso-Karamoja region, Uganda. J Ethnobiol Ethnomed. 2019;15:3 https:// doi.org/10.1186/s13002-018-0278-8.

110. Jafari-Sales A, Jafari B, Sayyahi J, Zohoori-Bonab T. Evaluation of antibacterial activity of ethanolic extract of Malva neglecta and Althaea officinalis L. on antibiotic-resistant strains of Staphylococcus aureus. J Biol Today's World. 2015;4(2):58-62. https://doi.org/10.15412/J.JBTW.01040205.

111. Tüzün BS, Hohman J, Kivcak B. Green Bio-inspired synthesis, characterization and activity of silver nanoparticle forms of Centaurea virgata Lam. and the isolated flavonoid eupatorin. Green Process Synth. 2008;7:372-9 https://doi. org/10.1515/gps-2017-0027.
112. Al KS. A survey of plants used in Jordanian traditional medicine. Int. j. pharmacogn. 1995;33(4):317-23.

113. Al-Douri NA. A survey of medicinal plants and their traditional uses in Iraq. Pharm. Biol. 2000;38:74-9 https://doi.org/10.1076/13880209(200001)3811-BFT074.

114. Oran SA. A list of flowering wild plants in Tafila Province, Jordan. Int J Biodivers Conserv. 2014;6(1):28-40 https://doi.org/10.5897/JJBC2011.116.

115. Polat R, Çakilcioğlu U, Satıl F. Traditional uses of medicinal plants in Solhan (Bingöl-Turkey). J Ethnopharmacol. 2013;148(3):951-63 https://doi.org/10. 1016/j.jep.2013.05.050

116. Said O, Khalil K, Fulder S, Azaizeh H. Ethnopharmacological survey of medicinal herbs in Israel, the Golan Heights, and the West Bank region. J Ethnopharmacol. 2002;83:251-65.

117. Khare CP. Indian medicinal plants - an illustrated dictionary. India, New Delhi: Springer Science and Business Media LLC; 2007. p. 49.

118. Ali-Shtayeh MS, Jamous RM, Al-Shafie' JH, Elgharaban WA, Kherfan AF, Qarariah HK, Khdair SI, Soos IM, Musleh AA, Isa BA, Herzallah HM, Khlaif RB, Aiash SM, Swaiti GM, Abuzahra MA, Haj-Ali MM, Saifi NA, Azem HK, Nasrallah HA. Traditional knowledge of wild edible plants used in Palestine (Northern West Bank): A comparative study. J Ethnobiol Ethnomed. 2008;4:1-13. https://doi.org/10.1186/1746-4269-4-13.

119. Karaaslan Ö, Cöteli $E$, Karataş F. Investigation of amounts of $A, E, C$ vitamins with malondialdehyde and glutathione in plant Gundelia tournefortii. Erzincan Üniv Fen Bilim Enst Derg. 2014;7-2:159-68 https://doi.org/10.18185/ eufbed. 44500

120. Mosaddegh M, Naghibi F, Moazzeni H, Pirani A, Esmaeile S. Ethnobotanica survey of herbal remedies traditionally used in Kohghiluyeh va Boyer Ahmad province of Iran. J Ethnopharmacol. 2012;141:80-95. https://doi.org/ 10.1016/j.jep.2012.02.004

121. Demir F, Özcan M. Chemical and technological properties of rose (Rosa canina L.) fruits grown wild in Turkey. J Food Eng. 2001;47(4):333-6 https:// doi.org/10.1016/S0260-8774(00)00129-1.

122. Keser S, Keser F, Kaygili Ö, Tekin S, Türkoğlu I, Demir E, Türkoğlu S, Karatepe M, Sandal S, Kirbağ S. Phytochemical compounds and biological activities of Celtis tournefortii fruits. Anal Chem Lett. 2017;7(3):344-55 https://doi.org/10. 1080/22297928.2017.1329664

123. Mason S, Nesbitt M. Acorns as food in southeast Turkey: Implications for prehistoric subsistence in southwest Asia. In: Fairbairn A, Weiss E, editors From foragers to farmers. Oxford: Oxbow Books; 2009. p. 71-85.

124. Spînu SS, Ortan A, Călin A, Moraru I, Ionescu D. Liquiritiae radix-a short review of its properties and applications. Res J Agric Sci. 2017;49(4):284.

125. Wetherilt H, Pala M. Herbs and spices indigenous to Turkey. In: Charalambous G, editor. Spices, herbs and edible fungi. Developments in food science, vol. 34. Amsterdam, The Netherlands: Elsevier; 1994. p. 285-307.

126. Rayne S, Mazza G. Biological activities of extracts from Sumac (Rhus spp.): A review. Plant Foods Hum Nutr. 2007:62:165-75.

127. Shabbir A. Rhus coriaria Linn, a plant of medicinal, nutritional and industrial importance: a review. J Anim Plant Sci. 2012;22:505-12.

128. Nocentini D, Pacini E, Guarnieri M, Nepi M. Flower morphology, nectar traits and pollinators of Cerinthe major (Boraginaceae-Lithospermeae). Flora. 2012 207:186-96. https://doi.org/10.1016/j.flora.2012.01.004.

129. Halder S, Sharma A. A review on Urtica dioica L. World J Pharm Pharm Sci. 2017;6(10):404-21. https://doi.org/10.20959/wjpps201710-10249.

130. Chrubasik C, Roufogalis BD, Müller-Ladner U, Chrubasik S. A systematic review on the Rosa canina effect and efficacy profiles. Phytother Res. 2008; 22(6):725-33. https://doi.org/10.1002/ptr.2400.

131. ISE/International Society of Ethnobiology 2006. The International Society of Ethnobiology Code of Ethics (with 2008 additions). http://www. ethnobiology.net/code-of-ethics/. Accesed 24 May 2019.

\section{Publisher's Note}

Springer Nature remains neutral with regard to jurisdictional claims in published maps and institutional affiliations. 